\title{
GESTIÓN INDIVIDUAL DEL EQUILIBRIO ENTRE EL TRABAJO Y LA VIDA PERSONAL: REVISIÓN E INTEGRACIÓN DE LA LITERATURA*
}

\author{
MARÍA CANDELA RODRÍGUEZ"“ \& GUILLERMO ENRIQUE DABOS \\ UNIVERSIDAD NACIONAL DEL CENTRO DE LA PROVINCIA DE BUENOS AIRES (ARGENTINA)
}

Recibido/ Received/Recebido: 23/09/2015-Aceptado/ Accepted / Aprovado: 01/07/2016

\section{Resumen}

La sociedad y el mercado laboral han sufrido importantes cambios en los últimos años que generaron conflictos entre la vida laboral y personal de los individuos, causando resultados negativos en los mismos (insatisfacción y estrés) y en las organizaciones (aumento de rotación y ausentismo). Actualmente la literatura no brinda conocimiento suficiente para orientar a empleadores y empleados sobre las acciones para evitar el conflicto trabajo-vida. El objetivo de este trabajo es integrar la teoría sobre estrategias individuales para lograr el equilibrio trabajo-vida, identificando sus efectos en los individuos y en la organización que los emplea. Para ello, se revisaron publicaciones en revistas prestigiosas del campo del Management, entre 2000-2015. Se concluye con una discusión sobre las implicancias para gestionar los Recursos Humanos.

Palabras clave: Conflicto; Equilibrio; Trabajo; Vida; Estrategias individuales; Gestión; Recursos humanos.

\section{INDIVIDUAL MANAGEMENT OF BALANCE BETWEEN WORK AND PERSONAL LIFE: REVIEW AND INTEGRATION OF LITERATURE}

\begin{abstract}
Society and the labor market have undergone significant changes in recent years that led to conflicts between work and personal lives of individuals, causing negative results in the individual (dissatisfaction and stress) and in organizations (increased turnover and absenteeism). Literature does not currently provide sufficient knowledge to guide employers and employees on actions
\end{abstract}

Artículo de revisión bibliográfica realizado en el marco del proyecto: "Nuevas Relaciones de Empleo en la Economía del Conocimiento: Implicancias a nivel individual, organizacional y de cluster regional", perteneciente al Centro de Estudios en Administración (CEA), Facultad de Ciencias Económicas, Universidad Nacional del Centro de la Provincia de Buenos Aires (UNICEN).

* Licenciada en Administración, Facultad de Ciencias Económicas, Universidad Nacional del Centro de la Provincia de Buenos Aires. Docente del área de Administración, e investigadora integrante del Centro de Estudios en Administración de la Facultad de Ciencias Económicas, Universidad Nacional del Centro de la Provincia de Buenos Aires. Dirección postal: Pinto 399, Universidad Nacional del Centro de la Provincia de Buenos Aires, Facultad de Ciencias Económicas, Tandil, Bs. As. (B7000GHG) Argentina. Teléfono: 54(249) 438-5580 Int. 4322. Correo electrónico: candela.rodriguez@econ.unicen.edu.ar

*.* Ph.D. in Groups, Organizational Effectiveness, and Technology, Carnegie Mellon University. Profesor Titular de Teoría de la Organización, Director de la Maestría en Administración de Negocios y del Doctorado en Administración de la Facultad de Ciencias Económicas de la UNICEN. Co-director del Centro de Estudios en Administración de la Facultad de Ciencias Económicas de la UNICEN. Profesor e investigador afiliado de la Universidad de San Andrés. Correo electrónico: gdabos@econ.unicen.edu.ar 
to avoid work-life conflict. The aim of this work is to integrate the theory of individual strategies to achieve work-life balance, identifying their effects on individuals and in the organization that employs them. For this purpose publications were reviewed from prestigious journals in the field of Management between 2000-2015. It concludes with a discussion about the implications for managing the Human Resources.

Keywords: Conflict; Balance; Work; Lifetime; Individual strategies; Management; Human resources.

\title{
GERENCIAMENTO INDIVIDUAL DO EQUILÍBRIO ENTRE O TRABALHO E A VIDA PESSOAL: REVISÃO E INTEGRAÇÃO DA LITERATURA
}

\begin{abstract}
Resumo
Nos últimos anos, a sociedade e o mercado laboral têm sofrido importantes mudanças geradoras de conflitos entre a vida laboral e pessoal dos indivíduos, causando resultados negativos nos mesmos (insatisfação e estresse) e nas organizações (aumento de rotação e faltas). Atualmente a literatura não oferece conhecimento suficiente para orientar empregadores e empregados sobre as ações para evitar o conflito trabalho-vida. O objetivo deste trabalho é integrar a teoria sobre estratégias individuais para conseguir o equilíbrio trabalho-vida, identificando seus efeitos nos indivíduos e na organização que os emprega. Para isso, se revisaram publicações em revistas prestigiosas do campo do Management, entre 2000-2015. Conclui-se com uma discussão sobre as implicações para gerenciar os Recursos Humanos.

Palavras chave: Conflito; Equilíbrio; Trabalho; Vida; Estratégias individuais; Gestão; Recursos humanos.

Rodríguez, M. \& Dabos, G. (2017). Gestión individual del equilibrio entre el trabajo y la vida personal: revisión e integración de la literatura. En: Revista de la Facultad de Ciencias Económica: Investigación y Reflexión. rev.fac.cienc.econ, XXV (1). DOI: http://dx.doi. org/10.18359/rfce.1055
\end{abstract}

JEL: J50, J59.

\section{Introducción}

En las últimas décadas, se han producido una serie de cambios importantes en la sociedad y particularmente en el mercado laboral, entre los que se destacan la incorporación de la mujer al mundo del trabajo (Jalilvand, 2000; Richie, 2006), los puestos laborales cada vez más complejos y demandantes (Perlow, 1998; Park, Fritz \& Jex, 2011), y el creciente impacto de las tecnologías de la comunicación y de la información sobre el desarrollo de las tareas (Major \& Germano, 2006; Lanaj, Johnson \& Barnes, 2014). En gran medida, estos cambios han generado conflictos entre la vida laboral y la vida personal en un amplio rango de trabajadores en la actualidad (Batt \& Valcour, 2003; Kreiner, Hollensbe \& Sheep, 2009; Mustafa \& Gold, 2013), como resultado directo de que los límites entre ambos dominios han comenzado a disiparse.

La definición más utilizada sobre el conflicto trabajovida ha sido la desarrollada por Kahn et al. (1964), que lo define como una forma de conflicto inter-roles donde las presiones de los dominios del trabajo y de la vida personal, en algún punto, son incompatibles entre sí. Este conflicto ha cobrado mayor rele- 
vancia en la actualidad ya que la literatura empírica lo relaciona con resultados negativos, tanto para el individuo como para la organización. En particular, los estudios realizados en las últimas décadas revelan que si un individuo no alcanza un adecuado equilibrio entre el trabajo y la vida personal, se producen conflictos entre ambos dominios que afectan el desempeño en el trabajo, a partir de la disminución del rendimiento a nivel individual (Allen, Herst, Bruck \& Sutton, 2000) y grupal (Bhave, Kramer \& Glomb, 2010), el aumento en los índices de rotación (Greenhaus, Parasuraman \& Collins, 2001; Post, DiTomaso, Farris \& Cordero, 2009) y de ausentismo (Goff, Mount \& Jamison, 1990), la insatisfacción laboral (Bruck, Allen \& Spector, 2002; Kreiner, 2006) y hasta el deterioro en la salud de los individuos (Frone, Russell \& Cooper, 1997), con frecuentes episodios de agotamiento y estrés (Berkman, Buxton, Ertel \& Okechukwu, 2010; Parasurman \& Simmers, 2001).

Gran parte de la literatura existente sobre el conflicto trabajo-vida adopta una perspectiva organizacional, al focalizarse en la identificación de aquellas políticas de conciliación que las organizaciones implementan para mitigar las consecuencias negativas del conflicto y lograr que sus empleados mejoren el nivel de equilibrio (Chinchilla, Poelmans \& León, 2003; Carlier, 2006; Shockley \& Allen, 2007; Poelmans \& Beham, 2008; Trapero, Sánchez \& De la Garza García, 2013). Otros estudios analizan variables que precipitan el conflicto trabajo-vida, como la personalidad perfeccionista (Mitchelson, 2009), la posición formal dentro de la organización (DiRenzo, Greenhaus \& Weer, 2011), o las diferencias demográficas (Eby, Casper, Lockwood, Bordeaux \& Brinley, 2005). Finalmente, el conflicto trabajo-vida también ha sido examinado en su rol como mediador, por ejemplo, en la relación entre la cultura organizacional percibida y el estrés (Mauno, Kinnunen \& Pyykkö, 2005), o entre la cantidad de horas trabajadas y la angustia psicológica experimentada (Halbesleben, Harvey \& Bolino, 2009). Estos estudios confirman el impacto negativo asociado a una creciente conflictividad entre ambos dominios.

Más recientemente, otras investigaciones comenzaron a adoptar una perspectiva individual procu- rando encontrar respuesta a cómo los individuos actúan proactivamente para alcanzar un adecuado equilibrio entre el trabajo y la vida personal (Kossek, Ruderman, Braddy \& Hannum, 2012; McDowall \& Lindsay, 2014; Sturges, 2012). A pesar del creciente interés generado en la literatura, todavía resulta insuficiente el conocimiento sistematizado sobre la existencia y eficacia de las estrategias utilizadas por los individuos para reducir el conflicto entre ambos dominios (Kreiner et al., 2009). Una revisión recientemente publicada realiza un primer intento para abordar el tópico (Allen, Cho \& Meier, 2014), al examinar los estudios empíricos que investigaron la delimitación de dominios entre el trabajo y la vida personal desde una perspectiva individual. Sin embargo, también resultan importantes otras contribuciones que extienden el enfoque teórico de la delimitación de dominios (Nippert-Eng, 1996), y que ayudan a la comprensión de las estrategias para la gestión individual del equilibrio trabajo-vida, particularmente aquellas resultantes de teorías profundamente arraigadas en el comportamiento organizacional como, por ejemplo, la teoría de la identidad social (social identity, Ashforth \& Mael, 1989) o del rediseño de puestos de trabajo (job crafting) (Wrzesniewski \& Dutton, 2001).

Con sustento en una revisión exhaustiva de la literatura empírica y de los distintos enfoques teóricos involucrados, el objetivo de este artículo es analizar y sistematizar las estrategias que utilizan los individuos para alcanzar un adecuado equilibrio entre el trabajo y la vida personal. En particular, la presente revisión bibliográfica comprende los principales hallazgos en materia de estrategias individuales para la gestión del equilibrio entre el trabajo y la vida personal, que fueron reportados en revistas científicas de alto impacto desde el año 2000 hasta el presente. Se identifican dos grandes dimensiones de estrategias para la gestión individual del equilibrio entre ambos dominios, compuestas en cada caso por una serie de tácticas específicas que facilitan su implementación práctica. También se sistematizan los principales resultados de esta revisión y las implicancias que las estrategias tienen tanto para el individuo como para la organización. 
El siguiente apartado presenta la metodología de esta revisión, incluyendo la identificación de los artículos empíricos y las categorías emergentes para el posterior análisis de los hallazgos y contribuciones. Posteriormente, se detallan sintéticamente las principales teorías que explican cómo los individuos logran el equilibrio entre el trabajo y la vida personal. Luego, se examinan las estrategias utilizadas por los individuos para lograr dicho equilibrio, conforme emergen de la literatura empírica. Finalmente, se analizan las implicancias que estas estrategias tienen para el logro del equilibrio trabajo-vida, sus antecedentes y consecuencias, tanto a nivel individual como organizacional y, con sustento en la revisión realizada, se plantean interrogantes para el futuro de la investigación.

\section{Metodología de la revisión}

\subsection{Identificación de los artículos empíricos}

La metodología utilizada para la realización de esta revisión consistió en identificar aquellos artículos de mayor relevancia publicados en la literatura desde el año 2000 hasta la actualidad en las bases de datos EBSCO, JSTOR, Science Direct, Wiley y Psyc$N E T$. La selección intencional de dicho período de tiempo se justifica por el surgimiento de una gran cantidad de estudios empíricos sobre equilibrio trabajo-vida desde la perspectiva individual, a partir del desarrollo de la teoría de delimitación de dominios (boundary/border theory) (Ashforth, Kreiner \& Fugate, 2000; Clark, 2000) basada en la perspectiva social cognitiva originada inicialmente por NippertEng (1996). Estas teorías desarrollaron una clasificación similar de estrategias individuales basadas en el mismo continuo de "integración/segmentación" de los dominios, reflejando la necesidad de unificar el conocimiento existente sobre la temática.

La elección de las revistas incluidas en esta revisión se realizó en base al ranking del Financial Times, que representa el listado de revistas de más alta relevancia en el área de Administración y Negocios como disciplina científica. También se incluyeron las revistas indexadas en el Journal Citation Reports, con un factor de impacto mayor a 1,70, aun cuan- do no estuvieran incluidas en el ranking del Financial Times.

Con respecto a la identificación de los artículos empíricos, el proceso consistió en recurrir primero a términos genéricos vinculados a la temática, para luego ir acotando las palabras clave hacia aquellas más específicamente asociadas a la gestión individual del equilibrio trabajo-vida. En primera instancia, con el objetivo de identificar artículos que analizaran la temática desde diferentes perspectivas teóricas, la búsqueda se inició con palabras clave de índole más general, como: work-life balance, work-life conflict, work-family balance, work-family conflict, work-home interface, work-nonwork balance, work-nonwork conflict, role conflict, work and family. En cada artículo que surgía de la búsqueda se analizaba el abstract para determinar la pertinencia del mismo en relación al objetivo de esta revisión. En segunda instancia, con el objetivo de acotar aún más la búsqueda, se recurrió a términos más directamente asociados a la gestión individual del equilibrio trabajo-vida como: individual strategies, individual tactics, coping strategies, quality of life. En la última iteración, se utilizaron términos resultantes de las teorías involucradas como: role transition, role segmentation, role integration, role conflict, role identity, job crafting, boundary work, boundary work tactics.

Es importante destacar que de los artículos identificados se seleccionaron solo aquellos que tenían la particularidad de contener las palabras utilizadas para la búsqueda en el título, abstract o palabras claves. Asimismo, para que el trabajo fuera incluido en esta revisión, el objetivo del mismo debía estar relacionado con el logro del equilibrio trabajo-vida o la mejora de la calidad de vida desde una perspectiva individual o, alternativamente, vincular teorías sobre estrategias individuales con otras variables individuales $u$ organizacionales. Una vez finalizado el proceso de búsqueda, los estudios empíricos resultantes, y que cumplían con las condiciones de límite anteriormente establecidas, fueron 50 . En la Tabla 1 se puede observar la cantidad de trabajos identificados de cada revista, como también el enfoque metodológico de las investigaciones empíricas seleccionadas (38 trabajos cuantitativos y 12 cualitativos). 
Tabla 1: Trabajos identificados por revista y metodología utilizada

\begin{tabular}{|c|c|c|c|c|}
\hline Revistas & Cantidad & $\%$ & Cuantitativos & Cualitativos \\
\hline $\begin{array}{l}\text { Academy of Mana- } \\
\text { gement Journal }\end{array}$ & 5 & $10 \%$ & 2 & 3 \\
\hline Human Relations $^{1}$ & 7 & $14 \%$ & 2 & 5 \\
\hline $\begin{array}{l}\text { Human Resource } \\
\text { Management }\end{array}$ & 2 & $4 \%$ & 1 & 1 \\
\hline $\begin{array}{l}\text { Human Resource } \\
\text { Management Journal }\end{array}$ & 3 & $6 \%$ & 1 & 2 \\
\hline $\begin{array}{l}\text { Journal of Applied } \\
\text { Psychology }\end{array}$ & 2 & $4 \%$ & 2 & - \\
\hline $\begin{array}{l}\text { Journal of Business } \\
\text { and Psychology }{ }^{1}\end{array}$ & 4 & $8 \%$ & 4 & - \\
\hline $\begin{array}{l}\text { Journal of Occupa- } \\
\text { tional and Organiza- } \\
\text { tional Psychology }\end{array}$ & 2 & $4 \%$ & 2 & - \\
\hline $\begin{array}{l}\text { Journal of } \\
\text { Occupational Health } \\
\text { Psychology }\end{array}$ & 7 & $14 \%$ & 7 & - \\
\hline $\begin{array}{l}\text { Journal of Organiza- } \\
\text { tional Behavior }\end{array}$ & 4 & $8 \%$ & 4 & - \\
\hline $\begin{array}{l}\text { Journal of Vocational } \\
\text { Behavior }\end{array}$ & 10 & $20 \%$ & 9 & 1 \\
\hline Organization Science & 1 & $2 \%$ & 1 & - \\
\hline Personnel Psychology & 3 & $6 \%$ & 3 & - \\
\hline Total & 50 & $100 \%$ & 38 & 12 \\
\hline
\end{tabular}

Fuente: Elaboración propia.

\subsection{Identificación de categorías emergentes}

Una vez seleccionados los artículos empíricos que cumplen con las condiciones de límite fijadas para esta revisión, se realizó una clasificación de contenidos para facilitar el análisis de sus hallazgos y contribuciones. Para ello, se procuró dar respuesta a las siguientes preguntas: (i) ¿qué teorías se utilizaron para examinar cómo los individuos logran el equilibrio entre el trabajo y la vida personal?, (ii) ¿cuáles son las estrategias, emergentes de los artículos

$1 \quad$ Los trabajos de Mickel \& Dallimore (2009) y de McDowall \& Lindsay (2014) utilizan metodologías mixtas, pero para esta revisión se los considera cualitativos por el mayor desarrollo que presenta esta metodología en ambos artículos. empíricos seleccionados, que utilizan los individuos para alcanzar ese equilibrio?, y (iii) ¿qué implicancias tiene la aplicación de estas estrategias individuales en el logro efectivo del equilibrio trabajo-vida, tanto para el propio individuo como para la organización? A partir de estos interrogantes, surgieron las tres categorías que permiten estructurar los contenidos de esta revisión y analizar los hallazgos resultantes de la literatura empírica: (i) enfoques teóricos utilizados para abordar la temática, (ii) tipologías de estrategias individuales para lograr el equilibrio trabajo-vida, y (iii) implicancias de la gestión individual del equilibrio trabajo-vida.

\section{Revisión bibliográfica}

\subsection{Enfoques teóricos utilizados}

Durante el período que abarca esta revisión, distintos enfoques teóricos se han utilizado para lograr un mayor entendimiento sobre cómo el individuo puede alcanzar un equilibrio adecuado entre las demandas del trabajo y de la vida personal. Entre ellas, se destaca la teoría de la identidad social (Ashforth \& Mael, 1989), y en particular, la construcción de identidad (identity work) (Snow \& Anderson, 1987). Esta teoría, desarrollada inicialmente para intentar responder al interrogante de quiénes somos, argumenta que los individuos tienen múltiples identidades que se exteriorizan a través de los diferentes roles que deben cumplir y los distintos contextos en los que deben actuar (Ashforth \& Johnson, 2001). Este enfoque ha sido aplicado a la construcción de identidad, a nivel individual y organizacional, como forma de explicar las estrategias utilizadas por los individuos para alcanzar el equilibrio trabajo-vida (Kreiner, Hollensbe \& Sheep, 2006; Wayne, Randel \& Stevens, 2006; Bulger, Matthews \& Hoffman, 2007).

Otro enfoque utilizado es la teoría de delimitación de dominios (boundary work) (Nippert-Eng, 1996). Esta teoría describe cómo los individuos se involucran en el esfuerzo de la construcción, mantenimiento y desmantelamiento de límites entre el trabajo y la vida personal, considerando no solo su propia percepción sobre los límites, sino también las percepciones que los terceros desarrollan sobre éstos. 
Bajo esta perspectiva, los límites entre el trabajo y la vida personal se encuentran en un continuo en el cual los dominios pueden encontrarse totalmente integrados, totalmente segmentados o en algún punto intermedio del continuo. Esta posición depende, en gran medida, de la flexibilidad y permeabilidad de los límites (Ashforth et al., 2000; Clark, 2000; Hall Richter, 1988; Pleck, 1977). La flexibilidad se relaciona con la capacidad para expandir, contraer o acomodar los límites físicos y temporales de modo de poder atender las demandas de un dominio u otro; mientras que la permeabilidad se refiere al grado en que el individuo acepta que los aspectos psicológicos o comportamentales propios de un determinado dominio se entremezclen con el otro (Ashforth et al., 2000; Clark, 2000). De esta manera, los individuos suelen desarrollar distintas estrategias (de integración o segmentación) para gestionar los límites entre vida laboral y vida personal como mecanismo para alcanzar el equilibrio (Kreiner et al., 2009; Liu, Kwan, Lee \& Hui, 2013; Derks, Bakker, Peters \& van Wingerden, 2016).

Por otro lado, la teoría de gestión de la vida (life management theory of selection, optimization and compensation - SOC) (Baltes, 1997), establece un modelo basado en el desarrollo de una estrategia general de gestión de la vida, con el objetivo de lograr un balance positivo entre las ganancias y las pérdidas de recursos. Esta estrategia está asociada a la adopción de un conjunto específico de objetivos de desarrollo condicionados por las limitaciones que experimentan los individuos en la gestión de su vida (comportamiento de selección), al mantenimiento de aquellos recursos que específicamente contribuyen a los objetivos fijados (comportamiento de optimización), y al establecimiento de planes alternativos cuando es necesario contrarrestar la pérdida de recursos (comportamiento de compensación). Este enfoque sostiene que los individuos que utilizan esta estrategia de gestión a lo largo de su vida han demostrado, en general, ser más exitosos. Por ello, los autores que se basan en este enfoque lo extienden también al ámbito del conflicto trabajo-vida, indicando que estos comportamientos de selección, optimización y compensación también repercuten sobre ambos dominios $y$, en definitiva, sobre las probabilidades de alcanzar el equilibrio
(Baltes \& Heydens-Gahir, 2003; Young, Baltes \& Pratt, 2007; Baltes, Zhdanova \& Clark, 2011).

De la misma manera, la teoría sobre comportamientos proactivos de los empleados para rediseñar o moldear sus puestos de trabajo (job crafting) (Wrzesniewski \& Dutton, 2001) también se ha extendido al ámbito del conflicto trabajo-vida. Esta teoría analiza la iniciativa propia del individuo orientada a adaptar o cambiar cuestiones inherentes a sus puestos de trabajo con el fin de obtener un mayor control sobre el mismo y lograr su identidad en el lugar de trabajo. Particularmente, los individuos buscan cambiar o reinstaurar los límites de las relaciones que se establecen en un puesto de trabajo, así como también de las tareas que lo componen, para transformarlo en una experiencia más positiva y significativa. De esta manera, el conocimiento sobre el alcance de estos comportamientos o actividades no formales proporciona una sólida comprensión de los mecanismos alternativos para conciliar las demandas del trabajo y de la vida personal, constituyéndose en una premisa fundamental de la aplicación de este enfoque al logro del equilibrio trabajo-vida (Root \& Wooten, 2008; Sturges, 2012).

Otra perspectiva aplicada a la gestión del equilibrio trabajo-vida es la del proceso de toma de decisiones individuales, modelo que sostiene que las personas basan sus decisiones en una o más señales o piezas informativas, que luego son combinadas o recombinadas de alguna forma para llegar a sus decisiones (decision-making processes) (Ilgen, Major, Hollenbeck \& Sego, 1995). Greenhaus \& Powell (2003) analizaron el proceso de decisión de los individuos al momento de resolver el conflicto trabajo-vida, particularmente el generado por la imposibilidad de cumplir con las exigencias de ambos roles al mismo tiempo. Este enfoque evidencia que las personas basan sus decisiones en tres señales: (i) señales internas, que describen las prioridades del individuo en relación con el trabajo y la familia según el grado de identificación con cada uno; (ii) señales externas, que describen las prioridades de las otras personas involucradas en la situación; y por último, (iii) las señales de la función, que describen las características de las actividades involucradas. De esta manera, las decisiones individuales establecen estrategias que 
permiten gestionar el equilibrio trabajo-vida a través de la reprogramación de las actividades en función de las señales recibidas (Powell \& Greenhaus, 2006; Radcliffe \& Cassell, 2014).

Hasta la fecha, la teoría de delimitación de dominios ha sido la más relevante para el estudio del equilibrio trabajo-vida, ya que ha tenido mayor tiempo de desarrollo dentro de la temática. Por ello, no sorprende que la mayoría de las investigaciones empíricas examinadas hayan sido realizadas bajo esta perspectiva. Asimismo, la teoría de la construcción de identidad también tiene una amplia difusión y se encuentra sumamente relacionada con la teoría de delimitación de dominios al basarse en pilares similares (p. ej., el continuo de integración/segmentación). Finalmente, las otras tres teorías mencionadas en este apartado se han incorporado al estudio del equilibrio trabajo-vida recién en los últimos años, motivo por el cual evidencian un menor grado de desarrollo empírico dentro de la temática. Con sustento en estos enfoques teóricos, el siguiente apartado desarrolla una clasificación integradora de las estrategias individuales para la gestión del equilibrio trabajo-vida.

\subsection{Tipos de estrategias individuales}

La revisión de las investigaciones empíricas permite identificar dos grandes dimensiones de estrategias individuales para la gestión del equilibrio trabajovida. Estas dimensiones surgen del análisis de las diferentes clasificaciones desarrolladas por los distintos autores con sustento en los enfoques teóricos mencionados en el apartado anterior. Estas dimensiones están vinculadas a la formulación e implementación de la estrategia, de modo tal que, si una estrategia se diseña y se ejecuta exclusivamente por el individuo, se la incluye dentro de la dimensión de estrategias internas. En contraposición, si para ello se requiere la aprobación o la colaboración de terceros (p. ej., organización, compañeros de trabajo, familiares, amigos), se considera a esta dimensión como estrategias externas. Cabe destacar que cada una de estas dos estrategias se encuentra compuesta por tácticas específicas que permiten llevarlas a la práctica. Es decir, cada conjunto de tácticas específicas se relaciona con una dimensión estratégi- ca en particular. A continuación, se desarrollan las categorías de tácticas identificadas dentro de cada dimensión estratégica.

\subsection{Estrategias internas}

Dentro de estas estrategias se encuentran dos categorías de tácticas denominadas tangibles y actitudinales que, aunque puedan generar algún impacto sobre terceros, dependen únicamente de la decisión interna del individuo.

Las tácticas tangibles se refieren a aquellas que pueden ser percibidas de una manera precisa, siendo tácticas más visibles e identificables que las demás. En efecto, son tácticas relacionadas con la manipulación, tanto del espacio físico como de los artefactos o materiales, que ayudan a crear un mayor o menor distanciamiento entre los dominios del trabajo y de la vida personal, al establecer una clara distinción de límites (Kreiner et al., 2009; Sturges, 2012; Mustafa \& Gold, 2013; McDowall \& Lindsay, 2014). Por ejemplo, mudarse hacia una zona más lejana evidencia una táctica que manipula el espacio físico, como medio para ampliar la distancia entre la oficina y el hogar, probablemente con la intención de separar el trabajo de la vida personal. De igual modo, tomar la decisión de buscar trabajos que permitan la realización de tareas desde el hogar, implica una disminución de la distancia entre ambos dominios y denota una mayor preferencia por la integración.

En un estudio sobre jóvenes graduados universitarios, Sturges (2012) ofrece evidencia de la implementación de tácticas físicas que modifican el ámbito laboral, como tomar decisiones respecto qué tipo de tareas realizar o en qué tipo de proyectos involucrarse de modo de poder gestionar el tiempo dedicado a cada dominio en función del equilibrio buscado. De igual manera, McDowall \& Lindsay (2014), en su estudio sobre oficiales y administrativos de un departamento de policía, identificaron tácticas tangibles que requieren ajustes o cambios en el trabajo, como por ejemplo estructurar el espacio físico de la oficina para tener mayor control sobre el flujo de trabajo o programar las tareas para maximizar la eficiencia, siempre con vistas a conciliar las demandas procedentes del trabajo y de la vida personal. 
Otros estudios destacan la gestión de artefactos físicos, que implicaría, por ejemplo, utilizar llaveros diferentes para las llaves de cada dominio como señal de separación entre el trabajo y la vida personal o, en contraposición, mantener el correo electrónico laboral y personal dentro de una misma cuenta de mail como señal de integración (Kreiner et al., 2009). En este sentido, Mustafa \& Gold (2013) identificaron una serie de tácticas tangibles para individuos que realizan tareas laborales desde el hogar como la gestión de elementos físicos que delimitan un espacio de trabajo en una habitación, la afectación de una habitación exclusiva para la actividad laboral, o la utilización de una decoración diferente para el espacio destinado al trabajo en relación con el resto del hogar. En esta categoría, se encuentran también aquellas tácticas que implican la adopción de tecnología (Golden \& Geisler, 2007; Kreiner et al., 2009), como la utilización de teléfonos celulares o agendas electrónicas, para responder desde un mismo dispositivo a las demandas de ambos dominios, cuando se busca la integración entre ellos (D'Abate, 2005).

Una segunda tipología de tácticas son las actitudinales, que se vinculan con comportamientos que establecen formas de actuar y con la definición de las percepciones sobre el significado del equilibrio trabajo-vida para cada individuo. Por ejemplo, Baltes \& Heydens-Gahir (2003) usaron una base de datos on-line de empleados full-time para revelar que el uso de comportamientos de selección, optimización y compensación tiende a reducir los niveles de conflicto trabajo-vida. Los autores manifiestan que los comportamientos de selección hacen referencia al desarrollo, elaboración y compromiso con las metas $\mathrm{u}$ objetivos planteados respecto al equilibrio trabajo-vida, como por ejemplo, mantener totalmente separados los dominios del trabajo y del hogar. Los comportamientos de optimización consisten en adquirir, refinar, coordinar y aplicar los medios y recursos relevantes para lograr estas metas, como por ejemplo, establecer las tácticas físicas necesarias para impedir que el trabajo interfiera con las cuestiones personales. Los comportamientos de compensación se basan en el uso de mecanismos alternativos para mantener un nivel de rendimiento dado cuando los medios preexistentes han desa- parecido, como por ejemplo si resultara imposible cumplir con las tareas laborales dentro del horario de trabajo, establecer un lugar neutral para continuar el trabajo e impedir de esta forma realizar esas tareas compensatorias en el hogar. Se trata de un comportamiento de compensación que ayudaría al individuo a seguir manteniendo la separación entre ambos dominios, sin afectar el rendimiento laboral. Estos comportamientos implican actitudes y percepciones que permiten la flexibilidad para adaptarse a aquellos cambios que potencialmente pueden afectar el equilibrio.

Dentro de esta categoría, se identificaron también tácticas vinculadas con la construcción de la identidad para ayudar a negociar un equilibrio óptimo. Kreiner et al. (2006) realizaron un estudio sobre graduados universitarios donde encontraron que los individuos se involucran en actividades que separan el rol funcional de la identidad (lo que hago y lo que soy), que crean jerarquías entre identidades (asignando importancia a los roles), que ejecutan ocasionalmente roles efímeros y diferentes como vía de escape (asumiendo un tercer rol, distinto al laboral o al personal para descomprimir las presiones existentes), y finalmente tácticas que permiten elegir conscientemente qué identidad será explícita o implícita en un momento determinado (gestión de las identidades).

En este mismo sentido, Kreiner et al. (2006) y Mickel \& Dallimore (2009) coinciden con la importancia de establecer las prioridades de los roles que permitan al individuo adoptar una filosofía como guía para la toma de decisiones, percibiendo que no pierden nada a cambio o pensando en los beneficios potenciales que podrían acarrear en un futuro. Siguiendo esta línea, Kreiner et al. (2009) resaltan la necesidad de asignar un grado de urgencia a los problemas que se presentan en cada dominio, como medio para poder decidir de manera rápida y eficiente cuál es conveniente resolver primero. Las tácticas que implican la integración de las identidades se centran en fusionar conscientemente el rol con la identidad, como incorporar elementos únicos del ser a las tareas laborales o convertir el ser del individuo en un símbolo de la ocupación o de la ideología inherente a ella (Bulger et al., 2007). 
En este contexto, emergen también las estrategias híbridas que involucran actividades más neutrales entre la integración y la segmentación de dominios, como recargar energías desarrollando comportamientos que separan, de manera solo temporal, al individuo de las actividades propias de la ocupación, o alternativamente, la utilización de medios o recursos espirituales que ayuden a responder los dilemas de identidad (Kreiner et al., 2006).

También existe evidencia respecto al uso de tácticas que implican mantener en perspectiva lo que significan los dominios para cada individuo, como reconocer el esfuerzo que realizan en el trabajo y en su vida personal (el valor que aportan a cada dominio) o evitar sentimientos de culpabilidad por dedicarle mayor tiempo a un dominio que a otro (McDowall \& Lindsay, 2014). En este sentido, existe una necesidad de priorizar activamente el logro del equilibrio adecuado, teniendo una posición proactiva sobre la gestión del conflicto, pensando de manera positiva y siendo creativos en la identificación de eventuales soluciones. En esta línea, Kossek et al. (2012) resaltan la necesidad de identificar estilos particulares para gestionar el conflicto trabajo-vida que reflejan las preferencias de los individuos para el logro del equilibrio, dependiendo de la identificación con los roles y del control que perciben tener sobre los límites. Cada perfil identificado representa un conjunto de características psicológicas organizadas en distintas tácticas que demuestran un patrón de funcionamiento de los límites establecidos entre ambos dominios. En su estudio sobre gerentes, los autores identificaron seis perfiles de gestión de límites que determinan las tácticas a utilizar. Por ejemplo, los individuos que se caracterizan por tener un perfil orientado al trabajo, establecen estrategias que permiten la separación de los dominios favoreciendo las cuestiones laborales. En general, estos individuos presentan un bajo control sobre los límites, se encuentran altamente identificados con el rol del trabajo y tienden a permitir que las cuestiones laborales interfieran en el dominio de la vida personal.

Kreiner et al. (2009) también revelaron que los individuos utilizan tácticas actitudinales que permiten, en determinadas ocasiones, hacer más permeables los límites establecidos, para poder gestionar tem- poralmente los eventuales desbordamientos que se presentan entre un dominio y otro. Por ejemplo, atender una llamada personal en la oficina de manera excepcional implica la integración temporal de los dominios cuando la situación ineludiblemente lo amerita (Radcliffe \& Cassell, 2014), aun cuando se hayan adoptado estrategias explícitas que manifiestan una inclinación a evitar este tipo de interrupciones o desbordamientos entre dominios.

Finalmente, Mickel \& Dallimore (2009) encontraron tácticas actitudinales relacionadas con el establecimiento de prácticas continuas en trabajadores profesionales que influyen sobre la calidad de vida, entre las que se destacan, la adopción sistemática de actividades que mejoran la salud física o mental. En el mismo sentido, Michel, Bosch \& Rexroth (2014) manifiestan que los individuos que incorporan prácticas de atención plena (mindfulness) mejoran su distanciamiento psicológico del trabajo cuando están fuera del ámbito laboral, experimentando menos conflicto basado en tensión o agotamiento y aumentando la satisfacción con su equilibrio trabajo-vida.

\subsection{Estrategias externas}

Dentro de estas estrategias se encuentran dos categorías de tácticas implementadas por los individuos, temporales y relacionales. Como se mencionó anteriormente, para la aplicación de este tipo de tácticas se necesita de la colaboración de otros individuos o de la propia organización. Ambas generan consecuencias que impactan directamente sobre el individuo y sobre los demás actores involucrados.

Las tácticas temporales incluyen aquellas actividades que implican la gestión del tiempo que los individuos le dedican a los dominios del trabajo y la vida personal. Dentro de esta categoría, las tácticas se diseñan con el objetivo de poder controlar los horarios en ambos dominios, como manipular el tiempo dedicado al trabajo para poder maximizar las horas que se destinan al hogar (McDowall \& Lindsay, 2014), para incrementar la recreación o para realizar prácticas religiosas o espirituales (Mickel \& Dallimore, 2009). Otras tácticas similares implican encontrar tiempos para poder tomarse un respiro, gestionando descansos que permitan recuperarse 
de las demandas de ambos dominios (Kreiner et al., 2009; Mustafa \& Gold, 2013) o establecer una rutina horaria para realizar una actividad puntual previo a comenzar la jornada laboral (Sturges, 2012; Mustafa \& Gold, 2013). En tal sentido, los individuos utilizan esta actividad como tiempo de transición, que les permite la desconexión de las actividades personales y el inicio del "modo trabajo" (Mustafa \& Gold, 2013). Asimismo, los individuos también implementan otras tácticas para resolver el conflicto resultante de la superposición de actividades entre ambos dominios, recurriendo para ello a la reprogramación de actividades de modo de poder cumplir con todas ellas (Powell \& Greenhaus, 2006; Radcliffe \& Cassell, 2014).

Por otro lado, los resultados encontrados por Root \& Wooten (2008) en su estudio sobre empleados de una planta de autopartes, evidencian que los individuos incurren ocasionalmente en acciones disruptivas como respuesta a un ambiente de trabajo rígido. De esta manera, para lograr flexibilidad en el horario laboral y poder responder a las exigencias del dominio personal, los individuos suelen realizar acciones como justificar con mentiras la necesidad de llegar más tarde al trabajo o modificar la velocidad de las máquinas para poder finalizar la jornada, entre otras. La aplicación de este tipo de tácticas indudablemente puede implicar consecuencias en el desempeño individual y organizacional, en la calidad de la relación laboral con su empleador y en el nivel de descontento entre sus pares por la mayor cantidad de tareas que deben realizar.

La segunda categoría de tácticas se denominan relacionales, pues reúnen aquellas que implican una participación activa de otras personas, siendo la comunicación y los vínculos relacionales los pilares fundamentales para poder llevarlas a cabo. La construcción de un consenso es un aspecto central de las tácticas relacionales, mientras que en las temporales no se necesita generar un consenso entre las partes involucradas, aun cuando también puedan tener consecuencias sobre terceras personas. Por ejemplo, si el individuo manipula sus horarios laborales se produce un impacto directo en los compañeros de trabajo que deben reemplazarlo o realizar las tareas que no se cumplieron. En cambio, las tácticas relacionales funcionan en interacción con terceros, por ejemplo, establecer charlas con los compañeros de trabajo para hablar de temas personales si se busca la integración de ambos dominios. Esta táctica se utiliza como un medio para transmitir la importancia relativa que los individuos le dan a los roles del trabajo y la vida personal (D'Abate, 2005), o para establecer cuáles son las preferencias que cada individuo tiene en relación con el equilibrio que desea lograr (Kreiner et al., 2009; McDowall \& Lindsay, 2014).

Asimismo, resulta importante dentro de esta categoría hacer frente a los infractores, es decir, a aquellas personas que con sus acciones quebrantan las tácticas impuestas por los individuos (Kreiner et al., 2009; Trefalt, 2013). En este sentido, los individuos se involucran en la creación de reglas, que al ser transmitidas correctamente permiten evitar o controlar las acciones de los infractores (Mustafa \& Gold, 2013). Por el contrario, Trefalt (2013) en su estudio sobre una muestra de abogados, demostró la importancia de incorporar en el proceso de decisión de la gestión individual del equilibrio trabajovida a los actores que forman parte de los dominios. Específicamente, la autora demostró la ventaja que implica diseñar y poner en práctica tácticas en conjunto con los demás actores involucrados. De esta manera, se facilita la adopción de las tácticas por parte de los terceros involucrados, permitiendo tener en cuenta sus inquietudes y evitando el surgimiento de eventuales infractores.

Otra táctica relacional consiste en utilizar la disponibilidad y las habilidades de otras personas cercanas para que ayuden a gestionar situaciones en ambos dominios, por ejemplo, utilizar a la pareja para que intercepte consultas provenientes del trabajo o a la secretaria para que atienda las llamadas personales cuando el individuo está ocupado laboralmente (Kreiner et al., 2009). En tal sentido, también existe evidencia de tácticas que implican negociar con los compañeros de trabajo y supervisores para obtener el consentimiento, la ayuda o la flexibilidad necesaria para cumplir con los objetivos (Powell \& Greenhaus, 2006; Root \& Wooten, 2008; McDowall \& Lindsay, 2014; Radcliffe \& Cassell, 2014), por ejemplo, pedirle reemplazo a un compañero para 
poder responder a demandas provenientes del dominio personal. Por último, existe evidencia que los individuos se involucran en actividades de socialización con personas que comparten concepciones similares acerca del equilibrio trabajo-vida (Sturges, 2012; McDowall \& Lindsay, 2014). Por ejemplo, relacionarse con amigos que trabajan en una misma ocupación o profesión puede generar un vínculo que fomenta el equilibrio trabajo-vida, porque cada uno comprende mejor la situación en la que se encuentra el otro y aceptan ayudarse mutuamente toda vez que se generan episodios en donde se superponen las demandas provenientes de ambos dominios.

En la Tabla 2 se puede observar la clasificación de las estrategias individuales resultantes de la revisión de la literatura empírica. Es importante destacar que las tácticas desarrolladas en este apartado son complementarias entre sí, es decir, que un mismo comportamiento puede hacer referencia simultáneamente a dos tipos de tácticas. Realizar actividades que se establecen como rutinas (táctica actitudinal) e implementar rutinas para gestionar el tiempo de las transiciones (táctica temporal) pueden evidenciarse en un mismo comportamiento, como realizar actividad física antes del trabajo. En este sentido, las tácticas suelen ser multi-funcionales y se refuerzan entre sí (Kreiner et al. 2009). Asimismo, dependiendo de las preferencias del individuo para lograr el equilibrio trabajo-vida, estas estrategias pueden tener el fin de segmentar o de integrar ambos dominios entre sí, o alternativamente de lograr una combinación intermedia entre los extremos del continuo (Kreiner et al., 2006; Golden \& Geisler, 2007; Kreiner et al., 2009).

Tabla 2. Clasificación de las estrategias individuales de balance trabajo-vida

\begin{tabular}{|c|c|c|c|}
\hline \multicolumn{4}{|c|}{ Estrategias Individuales } \\
\hline \multicolumn{2}{|c|}{ Internas } & \multicolumn{2}{|c|}{ Externas } \\
\hline Tácticas Tangibles & Tácticas Actitudinales & Tácticas Temporales & Tácticas Relacionales \\
\hline $\begin{array}{l}\text { Adaptar límites físicos (Kreiner et } \\
\text { al., 2009; McDowall \& Lindsay, } \\
\text { 2014; Mustafa \& Gold, 2013) }\end{array}$ & $\begin{array}{l}\text { Utilizar comportamientos de selec- } \\
\text { ción, optimización y compensación } \\
\text { (Baltes \& Heydens-Gahir, 2003) }\end{array}$ & $\begin{array}{l}\text { Controlar el tiempo de trabajo (Mic- } \\
\text { kel \& Dallimore, 2009; Kreiner et al., } \\
\text { 2009; McDowall \& Lindsay, 2014) }\end{array}$ & $\begin{array}{l}\text { Conversar sobre la vida personal en e } \\
\text { trabajo (D'Abate, 2005) estableciendo } \\
\text { expectativas para su equilibrio trabajo- } \\
\text { vida (Kreiner et al., 2009; McDowall \& } \\
\text { Lindsay, 2014) }\end{array}$ \\
\hline $\begin{array}{l}\text { Manipular el espacio físico de tra- } \\
\text { bajo (Kreiner et al., 2009; Sturges, } \\
\text { 2012; Mustafa \& Gold, 2013) }\end{array}$ & $\begin{array}{l}\text { Gestionar las identidades (Kreiner } \\
\text { et al., 2006; Bulger et al., 2007) y } \\
\text { mantener la perspectiva sobre cada } \\
\text { dominios (McDowall \& Lindsay, } \\
\text { 2014) }\end{array}$ & $\begin{array}{l}\text { Encontrar un respiro de las responsa- } \\
\text { bilidades del trabajo y la vida personal } \\
\text { (Kreiner et al., 2009; Mustafa \& Gold, } \\
\text { 2013) }\end{array}$ & $\begin{array}{l}\text { Negociar con supervisores y compa- } \\
\text { ñeros de trabajo (Powell \& Greenhaus, } \\
\text { 2006; Root \& Wooten, 2008; McDowall } \\
\text { \& Lindsay, 2014) }\end{array}$ \\
\hline $\begin{array}{l}\text { Gestionar artefactos } 0 \text { materiales } \\
\text { físicos (Kreiner et al., 2009) }\end{array}$ & $\begin{array}{l}\text { Establecer prioridades asociadas a } \\
\text { cada rol (Kreiner et al., 2009, Mickel } \\
\text { \& Dallimore, 2009) }\end{array}$ & $\begin{array}{l}\text { Implementar rutinas para gestionar los } \\
\text { tiempo de las transiciones (Sturges, } \\
\text { 2012; Mustafa \& Gold, 2013) }\end{array}$ & $\begin{array}{l}\text { Hacer frente a eventuales infractores } \\
\text { (Kreiner et al., 2009), creando normas } \\
\text { (Mustafa \& Gold, 2013) }\end{array}$ \\
\hline $\begin{array}{l}\text { Utilizar la tecnología para integrar/ } \\
\text { separar dominios (Golden \& Geis- } \\
\text { ler, 2007; Kreiner et al., 2009; Mc- } \\
\text { Dowall \& Lindsay, 2014) }\end{array}$ & $\begin{array}{l}\text { Permitir la permeabilidad diferencial } \\
\text { de los límites (Kreiner et al., 2009; } \\
\text { Radcliffe \& Cassell, 2014) }\end{array}$ & $\begin{array}{l}\text { Reprogramar actividades para aten- } \\
\text { der demandas en ambos dominios } \\
\text { (Powell \& Greenhaus, 2006; Radcliffe } \\
\text { \& Cassell, 2014) }\end{array}$ & $\begin{array}{l}\text { Interactuar con otras personas para ob- } \\
\text { tener apoyo y consentimiento (Kreiner et } \\
\text { al., 2009; McDowall \& Lindsay, 2014; } \\
\text { Radcliffe \& Cassell, 2014) }\end{array}$ \\
\hline $\begin{array}{l}\text { Gestionar la cantidad o contenido } \\
\text { del trabajo a realizar (Sturges, 2012) }\end{array}$ & $\begin{array}{l}\text { Convertir prácticas en rutinas prio- } \\
\text { rizando el equilibrio trabajo-vida } \\
\text { (Mickel \& Dallimore, 2009; Mc- } \\
\text { Dowall \& Lindsay, 2014) } \\
\end{array}$ & $\begin{array}{l}\text { Flexibilizar el horario laboral tomando } \\
\text { acciones disruptivas (Root \& Wooten, } \\
\text { 2008) }\end{array}$ & $\begin{array}{l}\text { Socializar con personas que comparten } \\
\text { una concepción similar sobre el equili- } \\
\text { brio trabajo-vida (Sturges, 2012; Mc- } \\
\text { Dowall \& Lindsay, 2014) } \\
\end{array}$ \\
\hline $\begin{array}{l}\text { Cambiar de trabajo por uno que se } \\
\text { ajuste a las necesidades de equili- } \\
\text { brio trabajo-vida (McDowall \& Lind- } \\
\text { say, 2014) }\end{array}$ & $\begin{array}{l}\text { Practicar la atención plena (min- } \\
\text { dfulness) para mejorar el equilibrio } \\
\text { cognitivo-emocional entre dominios } \\
\text { (Michel et al., 2014) }\end{array}$ & & $\begin{array}{l}\text { Construir límites con los demás, tenien- } \\
\text { do en cuenta el impacto en las relacio- } \\
\text { nes y las propias reacciones del indivi- } \\
\text { duo (Trefalt, 2013) }\end{array}$ \\
\hline
\end{tabular}

Fuente: Elaboración propia. 


\subsection{Gestión individual del equilibrio trabajo- vida: hallazgos e implicancias}

La literatura ha examinado los efectos de la gestión individual del equilibrio trabajo-vida, a partir de la aplicación de estrategias y sus tácticas específicas para conciliar ambos dominios en relación con la eficacia en el logro de dicho equilibrio. También ha comenzado a examinar la influencia de variables que moderan o condicionan la relación entre la implementación de las estrategias individuales y el logro efectivo del equilibrio entre ambos dominios. Los hallazgos e implicancias resultantes de estas líneas de indagación se analizan en el presente apartado. Más allá del todavía incipiente desarrollo de la temática y de la escasa consolidación de este cuerpo de literatura empírica, los resultados alcanzados permiten identificar potenciales antecedentes y consecuencias de la gestión individual del equilibrio trabajo-vida, así como establecer relaciones significativas con otras variables relevantes del comportamiento organizacional. Este análisis concluye con un intento de sistematización de las principales variables y relaciones examinadas en los artículos empíricos identificados.

\subsection{Efectos de la gestión individual sobre el equilibrio trabajo-vida}

Con respecto al impacto que tiene la gestión individual en el equilibrio trabajo-vida, Olson-Buchanan \& Boswell (2006), en su estudio sobre empleados administrativos de una universidad pública, encontraron que el efecto de la implementación de estrategias de integración de los dominios sobre el equilibrio no es absoluto, sino que depende de la direccionalidad en la que se aplica la integración. Los individuos que prefieren integrar la vida personal al trabajo logran menor nivel de conflictividad, pero aquellos que prefieren integrar el trabajo a la vida personal tienden a incrementarlo. Asimismo, los individuos que implementan estrategias que implican una alta integración entre dominios, aunque sin direccionalidad alguna, presentan un grado de conflictividad muy alto como consecuencia de las continuas interferencias entre dominios (Kossek, Lautsch \& Eaton; 2006). Sin embargo, cabe destacar que la integración de dominios también manifiesta efectos positivos al permitir un cierto enriquecimiento entre los mismos, particularmente en la dirección del trabajo hacia la vida personal (McNall, Scott \& Nicklin, 2015). Muchas veces, por ejemplo, los individuos utilizan habilidades blandas aprendidas en el ámbito laboral para lidiar con los desacuerdos o intercambios de opiniones que se suscitan en el ámbito familiar.

Bulger et al. (2007), al estudiar una muestra de empleados en relación de dependencia, manifestaron la necesidad de entender los distintos aspectos de la gestión individual, a partir de la fortaleza de los límites establecidos entre ambos dominios, como determinante central que impacta en el logro efectivo del equilibrio trabajo-vida. Los autores encontraron que una escasa capacidad para flexibilizar los límites del trabajo (cuando los individuos no permiten que las cuestiones personales interfieran en su vida laboral) y una mayor permeabilidad del dominio de vida personal (cuando los individuos permiten que se produzcan desbordamientos psicológicos o comportamentales desde el dominio del trabajo), predicen la interferencia en la dirección del trabajo hacia la vida personal, pudiendo generar mayor conflictividad. Asimismo, es importante considerar que la disponibilidad para flexibilizar estos límites difiere entre hombres y mujeres. Las mujeres están más dispuestas a flexibilizar los límites del dominio del trabajo para permitir interferencias por parte del dominio de vida personal, y en cambio se muestran mucho menos dispuestas a aceptar que el desbordamiento suceda de la manera inversa (Matthews \& Barnes-Farrell, 2010). En esta misma línea, los individuos que tienen un bajo control sobre los límites establecidos en ambos dominios tienden a experimentar mayor conflictividad que aquellos con un alto control sobre los mismos (Kossek et al., 2012). Estos resultados ponen en evidencia la necesidad de indagar sobre el grado de control que ejercen los individuos sobre los límites que se establecen y sobre las diferencias de género con vistas a alcanzar un mayor entendimiento de los efectos de la gestión individual del equilibrio trabajo-vida.

Por otro lado, se ha estudiado la influencia que ejerce la identificación que tiene el individuo con los roles en la gestión de las estrategias individuales que implementan, generando impactos directos sobre 
el logro del equilibrio trabajo-vida. Cuando más se identifica el individuo con un determinado rol, es más probable que aplique estrategias individuales que integren ese rol a los otros dominios (Winkel \& Clayton, 2010) y que se encuentre menos dispuesto a abandonarlo (Matthews, Barnes-Farrell \& Bulger, 2010). Por ejemplo, si el individuo se identifica más con el rol del trabajo, probablemente permita que cuestiones inherentes al trabajo se filtren dentro del dominio de vida personal, como, por ejemplo, llevarse trabajo a la casa o atender llamadas del trabajo mientras se encuentra desempeñando actividades de su vida personal.

En efecto, existe evidencia empírica que muestra que los individuos gestionan activamente sus límites como forma de proteger su dominio preferido. Los individuos tienden a establecer estrategias que les permiten mantener separados ambos dominios, protegiendo uno de ellos, en particular, aquel que típicamente les reporta un mayor nivel de satisfacción y que les contribuye a lograr un mejor equilibrio (Methot \& LePine, 2016). De este modo, una alta identificación con un rol se relaciona con reacciones menos negativas a los desbordamientos indeseados hacia el otro rol, teniendo menos dificultad para realizar transiciones o concesiones en favor de aquel rol con el que se identifica más fuertemente (Hecht \& Allen, 2009). Sin embargo, Matthews et al. (2010) resaltan la importancia de gestionar cuidadosamente estas transiciones ya que su mayor frecuencia o repetitividad termina provocando una mayor integración entre dominios $y$, por ende, puede derivar en mayores niveles de conflicto particularmente en aquellos individuos que buscan mantener la separación entre los dominios del trabajo y la vida personal.

Asimismo, se ha encontrado una fuerte relación entre las parejas que comparten cuestiones laborales y el grado de preferencia para integrar ambos dominios. Específicamente, los esposos que comparten el lugar de trabajo o la ocupación, muestran mayor integración entre dominios que aquellos que no comparten aspecto laboral alguno (Halbesleben, Zellars, Carlson, Perrewe \& Rotondo, 2010; Methot \& LePine, 2016). Esta integración entre dominios genera que los roles, trabajos u ocupaciones com- partidas por los esposos influencien sobre la relación de pareja, que termina fluctuando entre el apoyo laboral que se brindan mutuamente y un cierto agotamiento emocional (Halbesleben et al., 2010). Siguiendo esta línea, si las preferencias del individuo $y$ de su pareja se encuentran alineadas, particularmente hacia la separación entre dominios, se logra cierto distanciamiento psicológico (psychological detachment) que ayuda a disminuir los niveles de estrés, fomentando el logro del equilibrio trabajovida (Hahn \& Dormann, 2013).

Por otro lado, el tipo de estrategia utilizada también influye en el estrés experimentado y en el distanciamiento psicológico del empleado cuando se encuentra fuera del horario laboral. En este sentido, las tácticas que implican el uso de artefactos tecnológicos para realizar actividades laborales desde el hogar están asociadas negativamente con el distanciamiento psicológico del trabajo fuera del horario laboral (Park et al. 2011; Derks, van Mierlo \& Schmitz, 2014), generando agotamiento en los individuos. Sin embargo, el uso de comunicaciones electrónicas, en particular, tiene diferentes efectos dependiendo del tono de la comunicación y del tiempo que requieran. Por ejemplo, cuando la comunicación se utiliza para proporcionar información positiva, existe un efecto favorable ya que provoca felicidad en los individuos. En cambio, para los individuos con mayor preferencia por la segmentación entre dominios, es más probable que experimenten una mayor conflictividad trabajo-vida, a medida que la comunicación electrónica requiera más tiempo de atención. En el caso de los individuos que prefieren la integración, no se evidencian efectos negativos por la exigencia de un mayor tiempo en la comunicación (Butts, Becker \& Boswell, 2015). Estos individuos admiten que el uso de dispositivos móviles para realizar comunicaciones puede ser útil para satisfacer simultáneamente demandas provenientes de ambos dominios, teniendo en consecuencia el potencial para reducir eventuales conflictos entre la vida laboral y la vida personal (Derks et al., 2016).

Por el contrario, las estrategias que implican la implementación de tácticas físicas predicen un cierto distanciamiento psicológico y una baja necesidad de 
recuperación, debido a que generan pausas temporales en las demandas laborales que previenen el agotamiento emocional (Sonnentag, Kuttler \& Fritz, 2010). No obstante, específicamente las tácticas físicas que generan un acercamiento entre la oficina y el hogar tienden a crear sentimientos de ansiedad, estrés y frustración en los individuos, demostrando que no todas las tácticas físicas impactan de la misma manera sobre el equilibrio trabajo-vida (Mustafa \& Gold, 2013).

En conclusión, la literatura empírica pone en evidencia la necesidad de estudiar el fenómeno teniendo en cuenta los diferentes aspectos que influyen en la gestión individual del equilibrio, como las características de las estrategias y tácticas utilizadas, la habilidad de los individuos para mantenerlas en el tiempo, sus preferencias respecto a la segmentación/integración de dominios, y otros factores que pueden ejercer influencia sobre la efectividad de las mismas. Si bien se ha logrado un cierto progreso en la comprensión del fenómeno, todavía se evidencia la necesidad de establecer resultados más firmes con respecto a la efectividad de la gestión individual en el logro del adecuado equilibrio entre el trabajo y la vida personal.

\subsection{Efectos moderadores}

Este apartado agrupa las investigaciones que indagaron el efecto de variables que moderan la relación entre la implementación de la gestión individual y el efectivo equilibrio trabajo-vida.

En particular, Young et al. (2007) demuestran que, en situaciones demandantes, el uso de tácticas de comportamientos SOC está relacionado con bajos niveles de estrés por factores externos. Los autores revelaron que el uso de estrategias SOC es más efectivo para individuos que tienen mayores demandas de recursos, particularmente para aquellos que se encuentran en mediana edad, con hijos pequeños, con bajo apoyo de su supervisor, o con escaso acceso a los beneficios de las políticas de conciliación trabajo-vida. Por otro lado, Baltes et al. (2011) en un estudio de trabajadores full-time con familia, indagaron el efecto del uso de estrategias SOC en la relación entre las características de la personalidad y el nivel de conflicto trabajo-vida experimentado. Sus resultados revelan que tanto la escrupulosidad como la afabilidad del individuo se relacionan con el uso de estrategias SOC, que influyen subsecuentemente en los niveles de conflicto trabajo-vida.

Asimismo, se han encontrado diferentes factores que influyen en las decisiones que toman los individuos para disminuir el conflicto trabajo-vida (Shockley \& Allen, 2015). En particular, las presiones derivadas del rol, el apoyo instrumental brindado por las personas que participan en cada rol, la importancia que le asignan los individuos a la actividad inherente al rol, el apoyo emocional recibido en el trabajo y la direccionalidad del conflicto trabajovida, predicen la decisión que toman los individuos respecto a la estrategia indicada para disminuir el conflicto experimentado. Otras variables que influencian estas decisiones se relacionan con la situación financiera del individuo y de su familia, con las consideraciones relativas a justicia y equidad, así como también con sus valores y creencias (Radcliffe \& Cassell, 2014).

Por otro lado, Matthews, Winkel \& Wayne (2014), en su estudio de naturaleza longitudinal sobre empleados full-time que experimentan sobrecarga de rol, encontraron que en el momento inmediato al evento, las transiciones entre dominios reducen la percepción de sobrecarga de rol en el individuo, al mismo tiempo que acrecientan su percepción sobre la conflictividad trabajo-vida. Es decir, cuando los individuos experimentan sobrecarga de rol, responden mediante la participación en mayores transiciones entre los roles, reasignando recursos desde un dominio (por ejemplo, el trabajo) hacia el dominio que se encuentra sobrecargado (por ejemplo, la vida personal). En consecuencia, debido a que el dominio sobrecargado elimina los escasos recursos del otro dominio, esta situación es percibida como un conflicto entre dominios. Los autores concluyen que estas estrategias se utilizan como medio para hacer frente al conflicto, pero que no constituyen estrategias preventivas que permitan disminuir la conflictividad en el tiempo.

Por último, una investigación reciente reveló que las normas sociales y el compromiso con la orga- 
nización son variables que moderan la relación entre el uso diario de smartphones para cuestiones laborales como estrategia individual y el conflicto trabajo-vida (Derks, Duin, Tims \& Bakker, 2015). Específicamente, los empleados que experimentan presiones normativas (de sus supervisores y compañeros de trabajo) para mantenerse comunicado fuera del horario laboral, tienen mayor conflictividad trabajo-vida en aquellos días que se enfrentan a un mayor uso de smartphones y sufren, por ende, mayor agotamiento laboral (Derks et al., 2014). Sin embargo, los empleados que están comprometidos con la organización moderan esta conflictividad. Estos hallazgos remarcan la necesidad de que aquellas organizaciones que intentan fomentar el uso de tecnologías de comunicación fuera del horario laboral, tengan en cuenta la importancia de lograr un elevado compromiso de sus empleados con la organización como forma de evitar un incremento en la conflictividad trabajo-vida. Asimismo, a nivel individual, los autores también demuestran los resultados negativos que puede generar el uso de esta táctica en particular.

Como se puede observar, las investigaciones sobre variables moderadoras que impactan en la relación entre las estrategias para la gestión individual y el efectivo equilibrio trabajo-vida aún son demasiado incipientes y se encuentran en proceso de desarrollo. Pocas investigaciones han examinado estos efectos moderadores, por lo que se evidencia la necesidad de incorporar en las futuras agendas de investigación el estudio sistemático de aquellas variables que moderan o condicionan el efecto de la gestión individual sobre el logro del efectivo equilibrio trabajo-vida.

\subsection{Antecedentes de la gestión individual del equilibrio trabajo-vida}

Con respecto a las variables que anteceden la gestión individual del equilibrio trabajo-vida, la literatura empírica ha abordado este fenómeno analizando diferentes variables inherentes al comportamiento organizacional y a la gestión de los recursos humanos, tanto desde el enfoque de las políticas de conciliación que implementa la organización como desde las percepciones individuales de apoyo recibi- do de parte de la organización o de los compañeros de trabajo. Estas variables afectan sobre la gestión individual.

En relación con las políticas de conciliación implementadas por las organizaciones, Kreiner (2006) en su estudio sobre graduados universitarios reveló que cuánto más cerca se encuentran las políticas organizacionales que ayudan a mantener separados los dominios a las preferencias de segmentación, en mejor posición se encontrarán los individuos para gestionar el equilibrio trabajo-vida como más les guste. Esta cercanía facilita la disminución del conflicto y del estrés experimentado por los individuos. En particular, estos individuos con alta congruencia entre sus preferencias y aquello ofrecido por la organización, experimentan menos conflicto trabajo-vida basado tanto en la gestión del tiempo (evitan demandas simultáneas de ambos dominios) como en la administración de las tensiones (evitan que las demandas de un dominio interfieran en el rendimiento del otro) (Chen, Powell \& Greenhaus, 2009).

No obstante, una determinada política de conciliación puede no ser beneficiosa para todos los empleados o generar inconvenientes en algunos grupos (Rothbard, Phillips \& Dumas, 2005). Específicamente, la incongruencia entre estas variables tiene menos efecto en los individuos que desean la integración comparado con aquellos que prefieren la separación entre dominios. Es decir, para los individuos que perciben un alto acceso a políticas integradoras, como onsite childcare (cuidado de los hijos en el lugar de trabajo), estas políticas generan una interacción más visible sobre las cuestiones inherentes a la vida personal en el ámbito de trabajo, provocando reacciones negativas en los empleados que prefieren la segmentación. En cambio, las políticas de flexibilidad horaria que tienden a fomentar la separación entre ambos dominios, pasan más desapercibidas por aquellos individuos que desean la integración, pues no generan influencia o interacción visible que pueda afectar su equilibrio trabajovida. Estos hallazgos sugieren que las políticas que promueven la separación evitan la incongruencia o malestar en los individuos que prefieren la integración, al tiempo que ayudan a quienes buscan 
una mayor segmentación entre ambos dominios (Rothbard et al., 2005).

Siguiendo esta línea, Shockley \& Allen (2010) en su estudio sobre empleados universitarios, encontraron que el uso de políticas de conciliación que brindan flexibilidad horaria y de lugar de trabajo se relaciona negativamente con la necesidad de separación entre dominios. Una variable que influye en esta relación es la responsabilidad familiar del individuo, evidenciando una necesidad de considerar al individuo y su entorno como impulsores del uso de políticas de conciliación flexibles. Asimismo, demostraron que las políticas de conciliación trabajovida no son utilizadas en forma igualitaria por todos los empleados, sino particularmente por aquellos que mantienen preferencias por la segmentación.

Asimismo, otros estudios destacan la importancia de brindar flexibilidad de lugar de trabajo como política de conciliación trabajo-vida en el marco de la gestión individual, ya que es un factor que condiciona la decisión sobre el tipo de estrategia que aplica el individuo para lograr su equilibrio (Radcliffe \& Cassell, 2014). En tal sentido, Lapierre, Steenbergen, Peeters \& Kluwer (2015) confirman la importancia de reconocer los problemas potenciales asociados a la gestión de límites de cuando existe la práctica del teletrabajo en las organizaciones. En efecto, cuando el teletrabajo es voluntario, los individuos pueden elegir qué grado de intensidad de teletrabajo se adapta mejor a las diversas demandas de sus roles. Sin embargo, cuando las organizaciones imponen una mayor integración física del trabajo y la vida personal, el desafío de crear y mantener límites entre ambos dominios resulta mucho más desafiante, sobre todo entre los empleados que tienen baja autoeficacia en el logro del equilibrio trabajo-vida. En este sentido, el teletrabajo puede no ser una solución adecuada para el conflicto trabajo-vida, especialmente en aquellos casos en que las organizaciones afectan la flexibilidad del teletrabajo, cuando deja de ser opción voluntaria para convertirse en una imposición a los individuos.

Otro antecedente importante en la gestión individual del equilibrio trabajo-vida es el apoyo percibido por los individuos en su ámbito de trabajo, tanto por parte de la organización como de sus compañeros de trabajo (Matthews et al., 2010; Ferguson, Carlson \& Kacmar, 2015). En particular, la percepción de normas compartidas por el grupo de trabajo que tienden a fomentar la segmentación entre dominios, está asociada positivamente con el distanciamiento psicológico del trabajo durante el horario no laboral, experimentando menor nivel de conflicto trabajo-vida. En tal sentido, los empleadores necesitan ser más sensibles sobre la necesidad que tienen sus empleados de lograr el distanciamiento psicológico adecuado para poder reducir los niveles de estrés como forma de implementar estrategias efectivas para la conciliación entre el trabajo y la vida personal (Park et al., 2011). Siguiendo esta línea, Koch \& Binnewies (2015) en un estudio sobre empleados y sus supervisores directos, revelan que los supervisores que son vistos como más amigables por sus empleados en cuestiones relativas al equilibrio trabajo-vida juegan un rol clave para fomentar los comportamientos de segmentación entre dominios y para establecer una cultura organizacional responsable que valore dicho equilibrio. Concretamente, aquellos empleados con supervisores que adoptan comportamientos orientados a la separación entre ambos dominios, son más propensos a segmentar el trabajo y la vida personal, logrando así sentirse menos agotados emocionalmente y más comprometidos con la organización.

Otro tipo de percepción individual que impacta como antecedente de la gestión individual del equilibrio trabajo-vida es el ostracismo laboral, que se refiere al grado en que un empleado percibe que es excluido o ignorado por sus compañeros de trabajo (Williams, 2001). Liu et al. (2013) en un estudio con empleados de una firma manufacturera, confirman que el ostracismo laboral está relacionado positivamente con el conflicto trabajo-vida y negativamente asociado con la satisfacción a nivel familiar. Asimismo, encontraron que la preferencia por la separación de ambos dominios disminuye el efecto mediador del conflicto trabajo-vida en la relación entre ostracismo y satisfacción laboral. Esto se debe principalmente a que los individuos con altos niveles de separación entre dominios tienen más probabilidades de poder reprimir los efectos del ostracismo laboral en el dominio de su vida personal, disminuyendo así el conflicto trabajo-vida y su efecto mediador como canalizador de la insatisfacción laboral. 
Con respecto a las implicancias de la satisfacción laboral en el equilibrio trabajo-vida, Ilies, Wilson \& Wagner (2009) en su estudio sobre empleados fulltime y sus parejas, demostraron que la satisfacción laboral diaria tiene un efecto importante en la satisfacción marital y en el estado de ánimo en el hogar. Asimismo, los autores concluyen que este efecto se ve incrementado en los individuos que prefieren la integración entre dominios, recomendando a las organizaciones que capaciten a sus empleados en cómo integrar sus roles para poder alcanzar un adecuado equilibrio entre el trabajo y la vida personal.

Por otro lado, también existe evidencia de que la carga laboral tiene un impacto significativo en la gestión individual del equilibrio trabajo-vida. En este sentido, Sonnentag et al. (2010) demostraron que la sobrecarga laboral está asociada positivamente con altos niveles de agotamiento emocional y con la necesidad del individuo de recuperarse, al mismo tiempo que se relaciona con un bajo nivel de distanciamiento psicológico del trabajo durante el horario no laboral. Estos resultados muestran que la sobrecarga laboral impide a los empleados establecer tácticas que les permitan lograr el distanciamiento psicológico necesario para poder recuperarse de las tensiones ocasionadas por el trabajo.

Otra de las variables que impacta como antecedente de la gestión individual del equilibrio trabajo-vida es el cambio organizacional, particularmente respecto al impacto que ejerce sobre la estabilidad de las estrategias utilizadas por el individuo (Ammons, 2013). En este sentido, el cambio en las normas de trabajo, particularmente respecto a cuándo y dónde se pueden realizar las tareas, no afecta a todos los individuos por igual. Bajo esta situación, los cambios en los límites pueden acelerarse cuando los empleados se sienten cómodos con el nuevo ambiente de trabajo, pero si esta situación no es acompañada simultáneamente por un cambio cultural en las normas familiares, las demandas del dominio personal podrían llegar a interferir y limitar las posibilidades reales de modificar las estrategias.

En síntesis, se puede destacar que estos resultados demuestran que la gestión del equilibrio entre el trabajo y la vida personal es un proceso complejo, donde diferentes variables tanto de nivel individual como organizacional emergen como antecedente. En tal sentido, se evidencia nuevamente el papel que cumple la organización en el logro efectivo del equilibrio trabajo-vida, demostrando que la misma no debe focalizarse solamente en la implementación de adecuadas políticas de conciliación, sino que también debe impulsar la adopción de estrategias tendientes a la gestión individual que impacten positivamente en el logro de un efectivo equilibrio entre el trabajo y la vida personal.

\subsection{Consecuencias de la gestión individual del equilibrio trabajo-vida}

Las investigaciones empíricas que han estudiado las consecuencias de la gestión individual del equilibrio trabajo-vida se focalizaron en diferentes características del fenómeno, como las preferencias por integrar o separar dominios, los tipos de estrategias y tácticas utilizadas, como así también sus características, con vistas a analizar sus efectos sobre determinadas variables de nivel tanto individual como organizacional, particularmente asociadas a la gestión estratégica de recursos humanos.

En este sentido, Rau \& Hyland (2002) en su estudio sobre estudiantes de postgrado a nivel de Maestría en Administración de Negocios, indagaron sobre la influencia de la percepción individual en el grado de conflicto trabajo-vida en sus búsquedas laborales. Sus resultados indican que los individuos que se encuentran buscando activamente trabajo $y$ presentan niveles altos de conflicto, perciben a las organizaciones con horario flexible y opciones de teletrabajo como más atractivas. Por lo general, los individuos que buscan la integración entre dominios tratan de facilitar las transiciones entre los roles, por lo que estos acuerdos de empleo flexibles facilitan el logro de sus objetivos. Por el contrario, aquellos individuos que necesitan separar los dominios para lograr un equilibrio adecuado, perciben a estos tipos de acuerdos de empleo como indeseables dado que facilitan una mayor integración entre los roles, provocando que los límites se desvanezcan. En este sentido, debido a que los individuos con una alta necesidad de separar los dominios prefieren mantener las tácticas físicas y temporales intactas, son menos 
propensos a percibir estos tipos de acuerdos como un medio viable para gestionar los límites entre ellos (Kossek et al, 2006; Shockley \& Allen, 2010).

La gestión individual del equilibrio trabajo-vida también impacta sobre el compromiso con la organización y la satisfacción laboral. En tal sentido, Rothbard et al. (2005) en su estudio sobre empleados universitarios, encontraron que aquellos individuos que prefieren separar los dominios tienen un menor compromiso con la organización y una menor satisfacción laboral, particularmente cuando ésta ofrece políticas de conciliación que facilitan la integración. Los autores también encontraron que esta situación se manifiesta incluso cuando la organización ofrece políticas que fomentan la separación de los dominios, llegando a la conclusión de los individuos que establecen estrategias que facilitan la segmentación del trabajo y la vida personal presentan, en general, menor nivel de compromiso organizacional. En esta línea, otro estudio confirma que los individuos que establecen estrategias para la gestión individual que producen una alta identificación con su rol en el trabajo, se encuentran más comprometidos y con menos intensiones de abandonar la organización (Wayne et al., 2006).

Scholarios \& Marks (2004), en un estudio sobre desarrolladores de software, demostraron el impacto de la flexibilidad brindada por el empleador para atender las demandas personales en las actitudes de los individuos. Los autores encontraron que la percepción de un trato justo sobre cuestiones que contribuyen a lograr el equilibrio trabajo-vida tiene un efecto significativo en la satisfacción laboral, en el compromiso organizacional y en la intención de permanecer en la organización. Sus resultados demuestran que, incluso en este tipo de industria donde los empleados tienen una inclinación individualista y con poca probabilidad de desarrollar lealtad a una única organización, se pueden generar ganancias para el empleado y el empleador mediante la implementación de prácticas activas que se preocupen por las actividades no laborales de los empleados. De esta manera, se genera un mayor compromiso con la organización permitiendo retener a los empleados y se contribuye a lograr el equilibrio deseado por el individuo como un objetivo secundario. Asimismo, existe evidencia empírica que muestra que es menos probable que los individuos abandonen la organización, cuando sus preferencias de equilibrio tienden a la integración, pues son más propensos a experimentar enriquecimiento entre ambos dominios y a evitar el agotamiento emocional (McNall et al., 2015). Las organizaciones que faciliten el desarrollo de preferencias integradoras en sus empleados y brinden más flexibilidad en la gestión del equilibrio trabajo-vida, se verán beneficiadas de estos resultados positivos.

De igual modo, la gestión individual del equilibrio trabajo-vida puede afectar el desarrollo de carrera y la estabilidad del contrato laboral. En este sentido, muchos individuos contemplan sus objetivos de carrera al momento de diseñar e implementar las estrategias de conciliación, como forma de evitar consecuencias negativas en su desarrollo de carrera (Sturges, 2008). Por ejemplo, los individuos que buscan establecerse en su carrera laboral o ascender rápidamente, modifican su gestión individual del equilibrio trabajo-vida, inclinándose hacia aquellas tácticas que favorecen las actividades laborales por encima de las personales. De esta manera, la gestión del equilibrio trabajo-vida puede convertirse en un comportamiento de autogestión de carrera, donde las consecuencias del logro del equilibrio entre estos dominios no impliquen un retraso o entorpecimiento en su desarrollo. Desde la perspectiva organizacional, los planes de carrera de cada empleado deberían tener en cuenta los objetivos relativos a la gestión individual del equilibrio trabajo-vida y, en particular, sus preferencias respecto a la integración/segmentación entre ambos dominios.

También es importante considerar que ocasionalmente, cuando los ambientes laborales son demasiado rígidos, los individuos suelen involucrarse en la adopción de estrategias que resultan disruptivas y que afectan el normal funcionamiento de la organización (Root \& Wooten, 2008). Los individuos que optan por estas acciones lo hacen incluso sabiendo que pueden poner en peligro la continuidad de su vínculo laboral con la organización (dependiendo de la gravedad de la acción disruptiva). Estos resultados evidencian la importancia atribuida por los individuos a la gestión del equilibrio trabajo-vida y las posibles consecuencias de su implementación tanto para la organización como para el propio individuo. 
Por último, cabe señalar que existe una cierta necesidad de adaptar las políticas de recursos humanos implementadas por las organizaciones a las necesidades y preferencias individuales para evitar resultados negativos. Es decir, el objetivo que deben perseguir las organizaciones para su gestión de recursos humanos es lograr una mejor integración entre las preferencias individuales y los beneficios ofrecidos por las mismas, como medio para que sus trabajadores puedan alcanzar un adecuado equilibrio entre el trabajo y la vida personal.

A modo de síntesis de la revisión y análisis realizado en esta sección, la Ilustración 1 sistematiza las principales variables y dimensiones resultantes de la investigación empírica sobre la gestión individual del equilibrio trabajo-vida. Con base en la relación principal entre las estrategias para la gestión individual y el efectivo equilibrio trabajo-vida alcanzado, se identifican las principales dimensiones de análisis y las variables moderadoras que influencian o condicionan esta relación. Asimismo, se identifican los principales antecedentes y consecuencias de la gestión individual del equilibrio trabajo-vida que han sido examinados, hasta la fecha, por la literatura empírica. Si bien el aún incipiente estado de desarrollo del tópico, no permite avanzar hacia una integración teórica más acabada, la Ilustración 1 contribuye a sistematizar las principales líneas de indagación, sus variables y dimensiones, que han venido guiando $y$ encaminando la investigación empírica sobre la gestión individual del equilibrio trabajo-vida.

Ilustración 1. Gestión individual del equilibrio trabajo-vida: variables y dimensiones emergentes de los artículos empíricos examinados

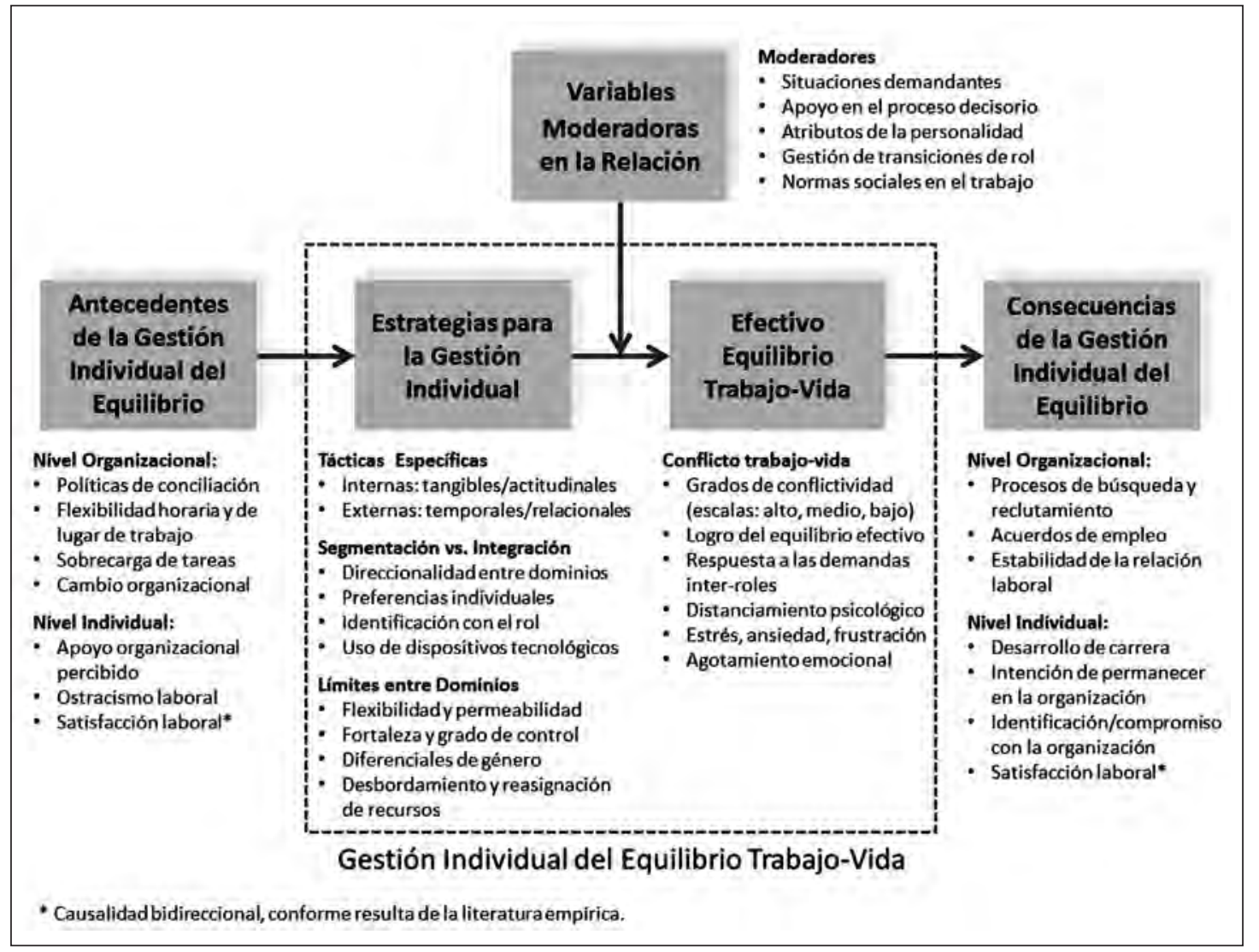

Fuente: Elaboración propia. 


\section{Discusión y conclusiones}

La gestión individual del equilibrio trabajo-vida implica el involucramiento del individuo en actividades que le permiten establecer el distanciamiento deseado entre los dominios, necesario para lograr el objetivo de conciliación planteado por el mismo. Como se expuso anteriormente, la gestión del equilibrio trabajo-vida no siempre es una decisión puramente individual, sino que existen estrategias que se co-construyen con la organización que los emplea y con las personas involucradas en cada uno de los dominios, ya sean compañeros de trabajo, jefes, familiares, amigos $e$ incluso clientes (Nippert-Eng, 1996; Ashforth et al., 2000; Halbesleben et al., 2010; Trefalt, 2013), dependiendo también de las condiciones laborales (Mustafa \& Gold, 2013). Como se pudo observar a lo largo de la revisión, los efectos de la implementación de la gestión individual en el logro de un efectivo equilibrio se encuentran menos desarrollados que los antecedentes y consecuencias del equilibrio trabajovida (en particular, respecto a las políticas y prácticas organizacionales de conciliación), poniendo en evidencia una necesidad de continuar en este camino para contribuir con la comprensión del fenómeno.

Desde una perspectiva organizacional, se puede señalar a las estrategias individuales como factores claves para el desarrollo de prácticas de gestión de recursos humanos que permitan enfrentar el desafío actual de atraer y retener a los empleados más valiosos en la organización (Cappelli, 2008). En este sentido, las estrategias externas representan una oportunidad para la implementación de políticas de conciliación, debido a que permiten conocer las preferencias de los individuos para lograr el equilibrio adecuado y participar en su implementación. Asimismo, en ausencia de políticas estandarizadas, las organizaciones también podrían favorecerse de estas estrategias fomentando la negociación de contratos idiosincráticos entre empleado y empleador (Rousseau, Ho \& Greenberg, 2006). Particularmente, se ha encontrado evidencia que destaca la negociación de términos idiosincráticos en los contratos laborales sobre aspectos relacionados con el equilibrio trabajo-vida, tales como la flexibilidad de los horarios (Dabos \& Rivero, 2012; Rousseau, Hornung \& Kim, 2009), de las tareas (Hornung,
Rousseau, Weigl, Müller \& Glaser, 2014), y del lugar de trabajo, (Rosen, Slater \& Johnson, 2013), como así también de términos que brindan apoyo en cuestiones personales (Ng \& Feldman, 2010).

En este sentido, el conocimiento de las preferencias de los individuos con respecto al equilibrio trabajovida y las estrategias que necesitan implementar, ayudaría a las organizaciones a establecer mejores prácticas para la gestión estratégica de los recursos humanos. Para ello resulta indispensable que investigaciones futuras avancen analizando cómo las organizaciones pueden contribuir con la implementación de estrategias externas. También se podría indagar sobre los factores necesarios para el establecimiento de las tácticas específicas, como el tipo de relación que debe existir entre el empleado y el empleador (leader-member exchange) para generar la confianza necesaria que permita llevar a cabo este tipo de negociaciones (Graen \& Uhl-Bien, 1995). Asimismo, al momento de formular planes estratégicos de recursos humanos destinados a la conciliación trabajo-vida de los empleados, las organizaciones deben tener en cuenta otras variables que también ejercen influencia en la gestión del equilibrio. Esta situación refleja la importancia de analizar en conjunto los diferentes niveles de análisis (esto es, individual, grupal y organizacional), como un paso ineludible para el desarrollo del tópico y de una comprensión multinivel sobre el equilibrio trabajo-vida (Ashforth et al., 2000; Kossek \& Lambert, 2005).

En la literatura académica, aún existe cierta falta de consenso acerca de las propiedades de determinadas políticas de conciliación trabajo-vida implementadas por las organizaciones, por ejemplo, la flexibilidad del horario laboral y del lugar de trabajo, particularmente en cuanto al grado de integración/segmentación de dominios que realmente proveen. Mientras que algunos autores sostienen que son políticas integradoras (Rau \& Hyland, 2002; Shockley \& Allen, 2010), otros sugieren que, en realidad, fomentan la segmentación de dominios (Rothbard et al., 2005). Futuras investigaciones deberían profundizar en la comprensión del impacto de las políticas de conciliación trabajo-vida que implementan las organizaciones respecto a las preferencias de los individuos (Shockley \& Allen, 2010), como así también seguir 
indagando sobre los antecedentes y consecuencias de la gestión individual del equilibrio trabajo-vida.

Desde una perspectiva individual, y más allá de la importancia de la intervención de otras personas en la gestión del equilibrio trabajo-vida, se observan escasos estudios que avanzan en esta línea de indagación. En efecto, Trefalt (2013) sostiene que solo considerando el contexto relacional de la gestión del equilibrio se puede contribuir al entendimiento de este fenómeno. Siguiendo esta línea, Mustafa \& Gold (2013) resaltan la influencia que el contexto ocupacional ejerce sobre la decisión de las estrategias a implementar por los individuos. Asimismo, futuras investigaciones deberían incorporar al análisis de aspectos interculturales (Ammons, 2013; Liu et al., 2013), como así también la etapa de carrera o de vida en la que se encuentra el individuo (Kossek et al., 2012; Hahn \& Dormann, 2013) para comprender mejor este fenómeno. Por otro lado, sería interesante indagar sobre el proceso de formación de estas estrategias individuales y sobre los factores que determinan las preferencias en materia de integración y segmentación.

Por otro lado, a nivel latinoamericano se puede evidenciar un avance en la incorporación de la temática en las agendas de investigación. En particular, existen estudios sobre los efectos negativos de la conflictividad trabajo-vida (Montenegro, 2007; Sánchez, Cegarra \& Cegarra, 2011), sobre políticas de conciliación implementadas por las organizaciones (Chinchilla et al., 2003; Carlier, 2006), como así también estudios que se focalizaron sobre las variaciones a nivel de género (Heller, 2013; Trapero et al., 2013) y a nivel generacional (Golik, 2013). Sin embargo, se evidencia una imperiosa necesidad de profundizar la indagación a nivel individual en Latinoamérica, ya que no existen a la fecha estudios empíricos que se focalicen en las estrategias implementadas por los individuos para lograr el equilibrio entre el trabajo y la vida personal. Futuras investigaciones podrían indagar sobre las estrategias que utilizan los individuos para conciliar ambos dominios en el contexto latinoamericano y estimular, de este modo, estudios comparativos entre culturas distintas que permitan identificar variaciones tanto en su implementación como en sus impactos a nivel individual y organizacional.
Por último, a nivel metodológico, continúa evidenciándose una inclinación hacia trabajos empíricos basados en métodos cuantitativos para el estudio de la interface trabajo-vida, en comparación con los cualitativos (Eby et al., 2005). Aunque se observa un aumento de estudios cualitativos (un $24 \%$ de los estudios incluidos en la presente revisión), resulta importante seguir fomentando el uso de esta metodología para posibilitar un mejor entendimiento del proceso de la gestión individual del equilibrio trabajo-vida. De la misma manera, también se evidencia la necesidad de incorporar estudios de naturaleza longitudinal, que permitan evaluar la estabilidad de las estrategias en el tiempo (Voydanoff, 2007; Kossek et al., 2012) y de utilizar muestras con características diferentes (Sturges, 2012). A pesar del gran número de estudios cuantitativos, se observa que solo cuatro investigaciones analizadas en esta revisión presentan como resultados escalas validadas para el estudio de la gestión del equilibrio trabajovida (Hecht \& Allen, 2009; Matthews \& BarnesFarrell, 2010; Matthews et al., 2010; Kossek et al., 2012). En este sentido, futuras investigaciones debieran avanzar en el desarrollo de instrumentos de medición de las estrategias individuales para poder analizar antecedentes y consecuencias de su implementación que permitan lograr simultáneamente mayor validación metodológica y mayor comprensión sustantiva de este fenómeno de creciente interés para la gestión de las organizaciones actuales.

\section{Referencias}

Allen, T., Cho, E. \& Meier, L. (2014). Work-family boundary dynamics. Annual Review of Organizational Psychology and Organizational Behavior, 1(1), 99-121.

Allen, T., Herst, D., Bruck, C. \& Sutton, M. (2000). Consequences associated with work-to-family conflict: A review and agenda for future research. Journal of Occupational Health Psychology, 5(2), 278-308.

Ammons, S. (2013). Work-family boundary strategies: Stability and alignment between preferred and enacted boundaries. Journal of Vocational Behavior, 82(1), 49-58.

Ashforth, B. \& Johnson, S. (2001). Which hat to wear? The relative salience of multiple identities in organizational contexts. En M. A. Hogg y D. J. Terry (Eds.), Social identity processes in organizational contexts. 31-48. Nueva York: The Haworth Press.

Ashforth, B., Kreiner, G. \& Fugate, M. (2000). All in a day's work: Boundaries and micro role transitions. Academy of Management Review, 25(3), 472-491. 
Ashforth, B. \& Mael, F. (1989). Social identity theory and the organization. Academy of Management Review, 14(1), 20-39.

Baltes, P. (1997). On the incomplete architecture of human ontogeny: Selection, optimization, and compensation as foundation of developmental theory. American Psychologist, 52(4), 366-380.

Baltes, B. \& Heydens-Gahir, H. (2003). Reduction of work-family conflict through the use of selection, optimization, and compensation behaviors. Journal of Applied Psychology, 88(6), 1005-1018.

Baltes, B., Zhdanova, L. \& Clark, M. (2011). Examining the relationships between personality, coping strategies, and work-family conflict. Journal of Business and Psychology, 26(4), 517-530.

Batt, R. \& Valcour, P. (2003). Human resources practices as predictors of work-family outcomes and employee turnover. Industrial Relations: A Journal of Economy and Society, 42(2), 189-220.

Berkman, L., Buxton, O., Ertel K. \& Okechukwu, C. (2010). Managers' practices related to work-family balance predict employee cardiovascular risk and sleep duration in extended care settings. Journal of Occupational Health Psychology, 15(3), 316-329.

Bhave, D., Kramer, A. \& Glomb, T. (2010). Work-family conflict in work groups: Social information processing, support, and demographic dissimilarity. Journal of Applied Psychology, 95(1), 145-158.

Bruck, C., Allen, T. \& Spector, P. (2002). The relation between work-family conflict and job satisfaction: A finer-grained analysis. Journal of Vocational Behavior, 60(3), 336-353.

Bulger, C., Matthews, R. \& Hoffman, M. (2007). Work and personal life boundary management: Boundary strength, work/personal life balance, and the segmentation-integration continuum. Journal of Occupational Health Psychology, 12(4), 365-375.

Butts, M., Becker, W. \& Boswell, W. (2015). Hot buttons and time sinks: The effects of electronic communication during nonwork time on emotions and work-nonwork conflict. Academy of Management Journal, 58(3), 763-788.

Cappelli, P. (2008). Talent on demand. Managing talent in an age of uncertainty. Boston: Harvard Business School Press.

Carlier, S. (2006). Las políticas de conciliación trabajo-familia en las empresas colombianas. Estudios Gerenciales, 22(100), 49-70.

Chen, Z., Powell, G. \& Greenhaus, J. (2009). Work-to-family conflict, positive spillover, and boundary management: a person-environment fit approach. Journal of Vocational Behavior, 74(1), 82-93.

Chinchilla, M., Poelmans, S. \& León, C. (2003). Políticas de conciliación trabajo-familia en 150 empresas españolas (No. D/498). IESE Business School.

Clark, S. (2000). Work/family border theory: A new theory of work/family balance. Human Relations, 53(6), 747-770.

D'Abate, C. (2005). Working hard or hardly working: A study of individuals engaging in personal business on the job. Human Relations, 58(8), 1009-1032.

Dabos, G. \& Rivero, A. (2012). Contratos idiosincráticos en la atracción y retención del talento: Tres estudios en organizaciones intensivas en conocimiento de la Argentina. Estudios Gerenciales, 28(125), 3-12.
Derks, D., Bakker, A., Peters, P. \& van Wingerden, P. (2016). Work-related smartphone use, work-family conflict and family role performance: The role of segmentation preference. Human Relations, 69(5), 1045-1068.

Derks, D., Duin, D., Tims, M. \& Bakker, A. (2015). Smartphone use and work-home interference: The moderating role of social norms and employee work engagement. Journal of Occupational and Organizational Psychology, 88(1), 155-177.

Derks, D., van Mierlo, H. \& Schmitz, E. (2014). A diary study on work-related smartphone use, psychological detachment and exhaustion: Examining the role of the perceived segmentation norm. Journal of Occupational Health Psychology, 19(1), 74-84.

DiRenzo, M., Greenhaus, J. \& Weer, C. (2011). Job level, demands, and resources as antecedents of work-family conflict. Journal of Vocational Behavior, 78(2), 305-314.

Eby, L., Casper, W., Lockwood, A., Bordeaux, C. \& Brinley, A. (2005). Work and family research in IO/OB: Content analysis and review of the literature. Journal of Vocational Behavior, 66(1), 124-197.

Ferguson, M., Carlson, D. \& Kacmar, K. (2015). Flexing work boundaries: The spillover and crossover of workplace support. Personnel Psychology, 68(3), 581-614.

Frone, M., Russell, M. \& Cooper, M. (1997). Relation of workfamily conflict to health outcomes: A four-year longitudinal study of employed parents. Journal of Occupational and Organizational Psychology, 70(4), 325-335.

Goff, S., Mount, M. \& Jamison, R. (1990). Employer supported child care, work/family conflict, and absenteeism: A field study. Personnel Psychology, 43(4), 793-809.

Golden, A. \& Geisler, C. (2007). Work-life boundary management and the personal digital assistant. Human Relations, 60(3), 519-551.

Golik, M. (2013). Las expectativas de equilibrio entre vida laboral y vida privada y las elecciones laborales de la nueva generación. Cuadernos de Administración, 26(46), 107-133.

Graen, G. \& Uhl-Bien, M. (1995). Relationship-based approach to leadership: Development of leader-member exchange (LMX) theory of leadership over 25 years: Applying a multi-level multidomain perspective. The Leadership Quarterly, 6(2), 219-247.

Greenhaus, J., Parasuraman, S. \& Collins, K. (2001). Career involvement and family involvement as moderators of relationships between work-family conflict and withdrawal from a profession. Journal of Occupational Health Psychology, 6(2), 91-100.

Greenhaus, J. \& Powell, G. (2003). When work and family collide: Deciding between competing role demands. Organizational Behavior and Human Decision Processes, 90(2), 291-303.

Hahn, V. \& Dormann, C. (2013). The role of partners and children for employees' psychological detachment from work and well-being. Journal of Applied Psychology, 98(1), 26-36.

Halbesleben, J., Harvey, J. \& Bolino, M. (2009). Too engaged? A conservation of resources view of the relationship between work engagement and work interference with family. Journal of Applied Psychology, 94(6), 1452-1465.

Halbesleben, J., Zellars, K., Carlson, D., Perrewé, P. \& Rotondo, D. (2010). The moderating effect of work-linked couple relationships and work-family integration on the spouse ins- 
trumental support-emotional exhaustion relationship. Journal of Occupational Health Psychology, 15(4), 371-387.

Hall, D. \& Richter, J. (1988). Balancing work life and home life: What can organizations do to help? Academy of Management Executive, 2(3), 213-223.

Hecht, T. \& Allen, N. (2009). A longitudinal examination of the work-nonwork boundary strength construct. Journal of Organizational Behavior, 30(7), 839-862.

Heller, L. (2013). Mujeres en la cumbre corporativa: el caso de la Argentina. Revista del Centro de Estudios de Sociología del Trabajo (CESOT), 1(3), 68-96.

Hornung, S., Rousseau, D., Weigl, M., Müller, A. \& Glaser, J. (2014). Redesigning work through idiosyncratic deals. European Journal of Work and Organizational Psychology, 23(4), 608-626.

Ilgen, D., Major, D., Hollenbeck, J. \& Sego, D. (1995). Raising an individual decision making model to the team level: A new research model and paradigm. En: R. A. Guzzo, et al. (Eds), Team effectiveness and decision making in organizations. 113-48. San Francisco, CA: Jossey-Bass.

Ilies, R., Wilson, K. \& Wagner, D. (2009). The spillover of daily job satisfaction onto employees' family lives: The facilitating role of work-family integration. Academy of Management Journal, 52(1), 87-102.

Jalilvand, M. (2000). Married women, work, and values. Monthly Labor Review, 123: 26-31.

Kahn, R.. Wolfe, D., Quinn, R., Snoek, J. \& Rosenthal, R. (1964). Organizational stress: Studies in role conflict and ambiguity. Oxford, England: Wiley.

Koch, A. \& Binnewies, C. (2015). Setting a good example: Supervisors as work-life-friendly role models within the context of boundary management. Journal of Occupational Health Psychology, 20(1), 82-92.

Kossek, E. \& Lambert, S. (2005). "Work-Family Scholarship": Voice and Context. Lawrence Erlbaum Associates Publishers.

Kossek, E., Lautsch, B. \& Eaton, S. (2006). Telecommuting, control, and boundary management: Correlates of policy use and practice, job control, and work-family effectiveness. Journal of Vocational Behavior, 68(2), 347-367.

Kossek, E., Ruderman, M., Braddy, P. \& Hannum, K. (2012). Worknonwork boundary management profiles: A person-centered approach. Journal of Vocational Behavior, 81(1), 112-128.

Kreiner, G. (2006). Consequences of work home segmentation or integration: A person environment fit perspective. Journal of Organizational Behavior, 27(4), 485-507.

Kreiner, G., Hollensbe, E. \& Sheep, M. (2006). Where is the "me" among the "we"? Identity work and the search for optimal balance. Academy of Management Journal, 49(5), 1031-1057.

Kreiner, G., Hollensbe, E. \& Sheep, M. (2009). Balancing borders and bridges: Negotiating the work-home interface via boundary work tactics. Academy of Management Journal, 52(4), 704-730.

Lanaj, K., Johnson, R. \& Barnes, C. (2014). Beginning the workday yet already depleted? Consequences of late-night smartphone use and sleep. Organizational Behavior and Human Decision Processes, 124(1), 11-23.
Lapierre, L., Steenbergen, E., Peeters, M. \& Kluwer, E. (2015). Juggling work and family responsibilities when involuntarily working more from home: A multiwave study of financial sales professionals. Journal of Organizational Behavior, 37(6), 804-822.

Liu, J., Kwan, H., Lee, C. \& Hui, C. (2013). Worl to family spillover effects of workplace ostracism: The role of work home segmentation preferences. Human Resource Management, 52(1), 75-93.

Major, D. \& Germano, L. (2006). The changing nature of work and its impact on the work-home interface. En: F. Jones, R. Burke \& M. Westman (Eds.), Work-life balance: A psychological perspective. 13-38. London: Psychology Press.

Matthews, R. \& Barnes-Farrell, J. (2010). Development and initial evaluation of an enhanced measure of boundary flexibility for the work and family domains. Journal of Occupational Health Psychology, 15(3), 330-346.

Matthews, R., Barnes-Farrell, J. \& Bulger, C. (2010). Advancing measurement of work and family domain boundary characteristics. Journal of Vocational Behavior, 77(3), 447-460.

Matthews, R., Winkel, D. \& Wayne, J. (2014). A longitudinal examination of role overload and work-family conflict: The mediating role of interdomain transitions. Journal of Organizational Behavior, 35(1), 72-91.

Mauno, S., Kinnunen, U. \& Pyykkö, M. (2005). Does work-family conflict mediate the relationship between work-family culture and self reported distress? Evidence from five Finnish organizations. Journal of Occupational and Organizational Psychology, 78(4), 509-530.

McDowall, A. \& Lindsay, A. (2014). Work-life balance in the police: The development of a self-management competency framework. Journal of Business and Psychology, 29(3), 397-411.

McNall, L., Scott, L. \& Nicklin, J. (2015). Do positive affectivity and boundary preferences matter for work-family enrichment? A study of human service workers. Journal of Occupational Health Psychology, 20(1), 93-104.

Methot, J. \& LePine, J. (2016). Too close for comfort? Investigating the nature and functioning of work and non-work role segmentation preferences. Journal of Business and Psychology, 31(1), 103-123.

Mitchelson, J. (2009). Seeking the perfect balance: Perfectionism and work-family conflict. Journal of Occupational and Organizational Psychology, 82(2), 349-367.

Michel, A., Bosch, C. \& Rexroth, M. (2014). Mindfulness as a cognitive-emotional segmentation strategy: An intervention promoting work-life balance. Journal of Occupational and Organizational Psychology, 87(4), 733-754.

Mickel, A. \& Dallimore, E.(2009). Life-quality decisions: Tensionmanagement strategies used by individuals when making tradeoffs. Human Relations, 62(5), 627-668.

Montenegro, G. (2007). La relación existente entre el conflicto trabajo-familia y el estrés individual en dos organizaciones colombianas. Cuadernos de Administración, 20(34), 139-160.

Mustafa, M. \& Gold, M. (2013). 'Chained to my work'? Strategies to manage temporal and physical boundaries among 
self employed teleworkers. Human Resource Management Journal, 23(4), 413-429.

$\mathrm{Ng}$, T. \& Feldman, D. (2010). Idiosyncratic deals and organizational commitment. Journal of Vocational Behavior, 76(3), 419-427.

Nippert-Eng, C. (1996). Home and work: Negotiating boundaries through everyday life. Chicago: University of Chicago Press.

Olson-Buchanan, J. \& Boswell, W. (2006). Blurring boundaries: Correlates of integration and segmentation between work and nonwork. Journal of Vocational Behavior, 68(3), 432-445.

Parasurman, S. \& Simmers, C. (2001). Type of employment, work-family conflict and well-being: A comparative study. Journal of Organizational Behavior, 22(5), 551-568.

Park, Y., Fritz, C. \& Jex, S. (2011). Relationships between workhome segmentation and psychological detachment from work: the role of communication technology use at home. Journal of Occupational Health Psychology, 16(4), 457-467.

Perlow, L. (1998). Boundary control: The social ordering of work and family time in high tech corporations. Administrative Science Quarterly, 43: 328-357.

Pleck, J. (1977). The work-family role system. Social Problems, 24: 417-427.

Poelmans, S. \& Beham, B. (2008). The moment of truth: Conceptualizing managerial work-life policy allowance decisions. Journal of Occupational and Organizational Psychology, 81(3), 393-410.

Post, C., DiTomaso, N., Farris, G. F. \& Cordero, R. (2009). Work-family conflict and turnover intentions among scientists and engineers working in R\&D. Journal of Business and Psychology, 24(1), 19-32.

Powell, G. \& Greenhaus, J. (2006). Managing incidents of work-family conflict: A decision-making perspective. Human Relations, 59(9), 1179-1212.

Radcliffe, L. \& Cassell, C. (2014). Resolving couples' work-family conflicts: The complexity of decision making and the introduction of a new framework. Human Relations, 67(7), 793-819.

Rau, B. \& Hyland, M. (2002). Role conflict and flexible work arrangements: The effects on applicant attraction. Personnel Psychology, 55(1), 111-136.

Richie, M. (2006). Demographic implications for work-family research. En: M. Pitt-Catsouphes, E. E. Kossek, \& S. Sweet (Eds.), The work and family handbook: Multidisciplinary perspectives, methods, and approaches. 125-140. Mahwah, NJ: Lawrence Erlbaum.

Root, L. \& Wooten, L. (2008). Time out for family: Shift work, fathers, and sports. Human Resource Management, 47(3), 481-499.

Rosen, C., Slater, D. \& Johnson, R. (2013). Let's make a deal development and validation of the ex post i-deals scale. Journal of Management, 39(3), 709-742.

Rothbard, N., Phillips, K. \& Dumas, T. (2005). Managing multiple roles: Work-family policies and individuals' desires for segmentation. Organization Science, 16(3), 243-258.

Rousseau, D., Ho, V. \& Greenberg, J. (2006). I-deals: Idiosyncratic terms in employment relationships. Academy of Management Review, 31(4), 977-994.
Rousseau, D., Hornung, S. \& Kim, T. (2009). Idiosyncratic deals: Testing propositions on timing, content, and the employment relationship. Journal of Vocational Behavior, 74(3), 338-348.

Sánchez, M. , Cegarra, D. \& Cegarra, J. (2011). ¿Influye el conflicto trabajo-vida personal de los empleados en la empresa. Universia Business Review, 29: 100-115.

Scholarios, D. \& Marks, A. (2004). Work life balance and the software worker. Human Resource Management Journal, 14(2), 54-74.

Shockley, K. \& Allen, T. (2007). When flexibility helps: Another look at the availability of flexible work arrangements and work-family conflict. Journal of Vocational Behavior, 71(3), 479-493.

Shockley, K. \& Allen, T. (2010). Investigating the missing link in flexible work arrangement utilization: An individual difference perspective. Journal of Vocational Behavior, 76(1), 131-142.

Shockley, K. \& Allen, T. (2015). Deciding between work and family: An episodic approach. Personnel Psychology, 68(2), 283-318.

Snow, D. \& Anderson, L. (1987). Identity work among the homeless: The verbal construction and avowal of personal identities. American Journal of Sociology, 92: 1336-1371.

Sonnentag, S., Kuttler, I. \& Fritz, C. (2010). Job stressors, emotional exhaustion, and need for recovery: A multi-source study on the benefits of psychological detachment. Journal of Vocational Behavior, 76(3), 355-365.

Sturges, J. (2008). All in a day's work? Career self management and the management of the boundary between work and non work. Human Resource Management Journal, 18(2), 118-134.

Sturges, J. (2012). Crafting a balance between work and home. Human Relations, 65(12), 1539-1559.

Trapero, F., Sánchez, L. \& De la Garza García, J. (2013). Políticas de diversidad y flexibilidad laboral en el marco de la responsabilidad social empresarial. Un análisis desde la perspectiva de género. Estudios Gerenciales, 29(127), 161-166.

Trefalt, S. (2013). Between you and me: Setting work-nonwork boundaries in the context of workplace relationships. Academy of Management Journal, 56(6), 1802-1829.

Voydanoff, P. (2007). Work, family, and community: Exploring interconnections. Mahwah, Nueva Jersey: Lawrence Erlbaum.

Wayne, J., Randel, A. \& Stevens, J. (2006). The role of identity and work-family support in work-family enrichment and its work-related consequences. Journal of Vocational Behavior, 69(3), 445-461.

Williams, K. (2001). Ostracism: The power of silence. New York: Guilford Press.

Winkel, D. \& Clayton, R. (2010). Transitioning between work and family roles as a function of boundary flexibility and role salience. Journal of Vocational Behavior, 76(2), 336-343.

Wrzesniewski, A. \& Dutton, J. (2001). Crafting a job: Revisioning employees as active crafters of their work. Academy of $\mathrm{Ma}$ nagement Review, 26(2), 179-201.

Young, L., Baltes, B. \& Pratt, A. (2007). Using selection, optimization, and compensation to reduce job/family stressors: Effective when it matters. Journal of Business and Psychology, 21(4), 511-539. 